\title{
Multimodale Evidentheitsmarkierung im Deutschen Eine Analyse verbaler und gestischer Ausdrucksformen und ihrer Kookkurrenzen*
}

Katinka Mangelschots (Leuven) und Steven Schoonjans (Innsbruck/Leuven)

\begin{abstract}
This study deals with gestures and their relation to verbal utterances in contexts of obviousness in German, focusing on debate situations. Obviousness is taken in a broad sense, referring to any state that is so evident or natural that no further proof is needed. The study offers an overview of the most common verbal and gestural markers of obviousness, including a brief discussion of combined uses of gestural markers. In a second step, the attention is shifted towards the interaction between these layers of expression (speech and gesture) by looking at the most frequent co-occurrence patterns of a verbal and a gestural marker.
\end{abstract}

\section{$1 \quad$ Einführung}

Everybody uses mime and gesture in real life, though we don't realize it. It's very useful as a performance technique, though it can be boring to watch on its own.

(Anthony Daniels)

Die Aussage von Anthony Daniels bringt es genau auf den Punkt: Gestik und Mimik sind ein inhärenter Teil von alltäglichen Gesprächen, aber diese Erkenntnis kommt erst, nachdem man auf sie aufmerksam gemacht wurde. Obwohl wir uns dieser Bewegungen zum Teil gar nicht bewusst sind, stellen sie sehr kräftige rhetorische Mechanismen dar. Sie verlieren aber teilweise ihre kräftige Wirkung und sogar einen großen Teil ihrer Bedeutung, wenn sie von der begleitenden verbalen Rede losgekoppelt werden (siehe 2.1). Das Diskussionsthema dieses Aufsatzes ist gerade diese Gestik, und insbesondere ihre Interaktion mit verbalen Evidentheitsmarkierern ${ }^{1}$ im Deutschen.

Im nächsten Abschnitt wird der theoretische Rahmen vorgestellt: Nach einer kurzen Darlegung des angewandten Gestikbegriffs (2.1) sowie der vertretenen Auffassung, was Evidentheit angeht (2.2), wird dargelegt, zu welchen Einsichten die bisherige Forschung der Kookkurrenz von gestischen und verbalen Evidentheitsmarkierern geführt hat (2.3). Der dritte Ab-

\footnotetext{
* Für relevante Anmerkungen zu früheren Fassungen des Beitrags danken wir Kurt Feyaerts, den Herausgebern von Linguistik Online und zwei anonymen Gutachtern.

1 Anstelle des gängigeren Begriffs ,Evidenz' ist in vorliegendem Beitrag von ,Evidentheit‘ die Rede, da ersterer Begriff, wie ein anonymer Gutachter anmerkt, auch auf Nachweise oder Indizien verweisen kann, was hier eindeutig nicht gemeint ist.
}

Linguistik online 81, 2/17 - http://dx.doi.org/10.13092/lo.81.3649

CC by 3.0 
schnitt liefert eine Beschreibung der Vorgehensweise und des Datenmaterials. Im Anschluss werden die Ergebnisse besprochen. Dabei wird zunächst auf die verbalen und die gestischen Evidentheitsmarkierer an sich eingegangen (4.1-2), bevor die Kookkurrenzmuster näher betrachtet werden (4.3). Diese Ergebnisse werden in Abschnitt 5 mit dem aktuellen Forschungsstand verglichen.

\section{Theoretischer Hintergrund}

\section{$2.1 \quad$ Gestik}

Eine Analyse der Kookkurrenz von Gesten und verbalen Evidentheitsmarkierern setzt eine Präzisierung der Begriffe ,Geste ' und ,Gestik' voraus. In jüngeren Zeiten wird zunehmend die Ansicht vertreten, dass Gespräche inhärent multimodal sind (z. B. Norris 2004: 1; Calbris 2011: xv). Bedeutung entsteht nicht nur auf der verbalen Ebene durch die Kombination von Wörtern: Unterschiedliche nonverbale Ebenen sind ebenso am Prozess beteiligt und es ist die Interaktion dieser unterschiedlichen Kommunikationsmodi, die einer Äußerung Bedeutung verleiht (vgl. auch Schoonjans 2014: 87-88).²

Im Folgenden wird auf nonverbaler Seite vor allem die Ebene der Gestik fokussiert. Als Gesten werden hier redebegleitende Körperbewegungen betrachtet, sofern sie einen Beitrag zur Kommunikation bzw. zur Vermittlung der intendierten Bedeutung leisten. Diese Auffassung ist mit Calbris' Definition von Gestik im Einklang:

the visible movement of any body part consciously or unconsciously made with the intention of communicating while speech is being produced.

(2011: 6)

Diese Definition beschränkt sich wohlgemerkt nicht auf redebegleitende Bewegungen der Hände und Arme (siehe u. a. McNeill 1992), sondern bezieht auch Kopf- und Schulterbewegungen mit ein.

\subsection{Evidentheit}

Da im Folgenden auf die Rolle von Gesten als Evidentheitsmarkierern eingegangen wird, ist eine kurze Erklärung des Evidentheitsbegriffes an dieser Stelle ebenso erforderlich. Eine eindeutige Abgrenzung dieses Begriffes zu liefern ist jedoch schwierig, da Evidentheit immer ein subjektives Phänomen ist. Zwar kann ein Sachverhalt als aus der Sicht des Sprechers evident oder aber als geradezu evident für alle dargestellt werden (vgl. Thurmairs 1989 Unterschied zwischen sprecher- und hörerbezogener Evidenz bei den Partikeln eben und einfach), aber die Einstufung als evident bleibt eine subjektive Entscheidung. Auch die Frage, wie breit oder wie eng ,Evidentheit' aufzufassen ist, ist nur schwer a priori zu beantworten. Diese Schwierigkeiten zeigen sich auch darin, dass auch in der bereits existierenden Literatur bislang noch kein richtiges Einverständnis im Hinblick auf die Definition von ,Evidentheit‘ bzw. ,Evidenz“

\footnotetext{
${ }^{2}$ Gelegentlich wird mit dem Begriff ,nonverbal` eine negative wissenschaftliche Auffassung gestischen und körperlichen Verhaltens verbunden. Diese Auffassung vertreten wir eindeutig nicht; der Begriff wird im vorliegenden Beitrag in rein denotativer Weise verwendet und verweist auf die Ausdrucksebenen, die nicht von Wörtern Gebrauch machen, ohne damit eine negative Bewertung oder eine hierarchische Herabstufung im Vergleich zur verbalen Ebene zu implizieren.
} 
erreicht werden konnte. Man denke zum Beispiel an die Frage, wie sich Evidentheit/Evidenz zu Evidentialität verhält: Während Forscher wie Bednarek (2006) die beiden Begriffe explizit miteinander in Verbindung setzen und Evidenz als einen Bereich der Evidentialität einstufen, betrachten andere (u. a. Dendale/Tasmowski 2001) die beiden Notionen als einander diametral entgegengesetzt. Diese Frage kann an dieser Stelle nicht endgültig beantwortet werden, zeigt aber die Schwierigkeiten bei der Abgrenzung des Evidentheitsbegriffs.

Wenn man den Begriff evident in großen deutschen Wörterbüchern wie Duden und Wahrig nachschlägt, findet man Verweise auf weitere Adjektive wie ,offenkundig', ,einleuchtend“ und ,keines Beweises bedürfend'. Ähnliche Umschreibungen liefern auch Bednarek (2006: 641) (,no evidence needed“) und Dendale/Tasmowski (2001: 340) (,rendering any further specification of its source [ $=$ the source of the information] or supporting evidence superfluous"). In diesen Definitionen von Bednarek und Dendale/Tasmowski wird allerdings vor allem die logische Natur des Sachverhalts fokussiert. Man kann jedoch den Begriff ,Evidentheit' auch weiter definieren, indem man ihn mit einem weiteren Zweig der Evidentialität verknüpft, nämlich mit ,sensory perception“ (Bednarek 2006: 640) bzw. mit der unbestreitbaren Sinneswahrnehmung (vgl. die in den bereits zitierten Wörterbüchern angesprochene Offenkundigkeit). Die Sinneswahrnehmung gilt mit anderen Worten als schlagender Beweis; weitere Beweise werden nicht gebraucht, wie es in der Definition des Wörterbuchs Le Grand Robert heißt:

Caractère de ce qui s'impose à l'esprit avec une telle force qu'il n'est besoin d'aucune autre preuve pour en connaître la vérité, la réalité.

(s. v. évidence; Hervorhebung von uns, KM/SS)

Noch expliziter werden die beiden möglichen Ebenen der Evidentheit (logische Natur oder unbestreitbare Sinneswahrnehmung) im Oxford English Dictionary online angegeben:

[T] he quality of being clearly perceptible; the fact, state, or condition of being easily seen or understood; plainness or openness to the eye or mind.

(s. v. obviousness)

Im Folgenden wird von dieser breiten Auffassung von Evidentheit ausgegangen. Festzuhalten ist somit, dass ein Sachverhalt evident ist bzw. sich als solcher darstellen lässt, wenn er leicht durchschaubar ist, weil er sich mit einer solchen Kraft dem Intellekt aufdrängt, dass kein zusätzlicher Beweis notwendig ist, um dessen Wahrheit bzw. Realität einzusehen - egal, ob diese Kraft der Logik oder der Wahrnehmung entstammt.

Der Bereich ist demnach nicht klar abzugrenzen, auch wenn die Definition so eindeutig aussehen darf. Diskurspartikeln beispielsweise bergen sehr viele Eigenschaften der Evidentheit in sich: 
1. $\quad$ Äußerung 2.23-26, 00:12:26.324-00:12:34.9153

Sido: Als ein Ausländer (-) öh hat er sich ja hervorragend integriert in dieses Land und er integriert ${ }^{\circ} \mathrm{hh} j a$ und ich bin $j a$ eigentlich wenn man so nimmt bin ich ja Deutscher ${ }^{\circ}$ hh $j a$

Dieser Ausschnitt enthält fünf Realisierungen von ja und gilt, der oben angeführten Definition von Evidentheit zufolge, als eine evidente Aussage: Es ist einsichtig, dass sich der Rapper Bushido, von dem hier die Rede ist, in Deutschland gut integriert hat (Bushido wird hier als ,Ausländer" bezeichnet, obwohl er in Deutschland geboren und aufgewachsen ist, allerdings als Sohn eines Tunesiers). Diese Aussage bedarf keiner weiteren Erörterung. Dass sein Kollege Sido, der hier spricht, Deutscher ist, dürfte den Gesprächspartnern und den Zuschauern ebenfalls, ohne weitere Ausführungen, bekannt und verständlich sein. Auch wenn ja nicht immer als reiner Evidentheitsmarkierer zu betrachten ist (besonders die zweite und die fünfte Realisierung des ja nähern sich vielmehr den Vergewisserungssignalen), bewirkt dieses gehäufte Vorkommen von ja eine Steigerung der Evidentheit.

Die im Folgenden vorzustellende Analyse stützt also auf einen breiten Evidentheitsbegriff, so wie er gerade definiert worden ist. Eine interessante Frage ist, inwiefern die Gestikanalyse zu einer Verfeinerung des Evidentheitsbegriffes (auch im Hinblick auf die verbale Ebene) führen könnte. Der Rahmen des vorliegenden Aufsatzes ist allerdings zu beschränkt, um diesem Ziel völlig gerecht werden zu können, sodass lediglich ein Anstoß in dieser Richtung geliefert werden kann.

Im Folgenden werden die Termini Evidentheit und Selbstverständlichkeit als Synonyme betrachtet und dementsprechend durcheinander verwendet.

\subsection{Evidentheit und Gestik in der Forschung}

Die Rolle der Gestik bei der Evidentheitsmarkierung ist bislang kaum erforscht. Zu nennen sind vor allem Untersuchungen, die sich zwar nicht explizit mit diesem Thema beschäftigen, es jedoch im Rahmen der Analyse berühren bzw. auf Gesten hinweisen, die vielleicht nicht selber Evidentheit im wahrsten Sinne des Wortes markieren aber in ihrer Verwendung durchaus häufiger mit verbalen Evidentheitsmarkierern einhergehen.

Whitehead (2011: 113) hat zum Beispiel darauf hingewiesen, dass das Kopfnicken (neben anderen Verwendungen, u. a. als Markierer von Einverständnis mit dem Gegenüber) andeuten kann, dass eine affirmierende Reaktion vonseiten des Gegenübers erwartet wird. Zwar dürfte es sich hier nicht um Evidentheit im wahrsten Sinne des Wortes handeln, aber wie Schoonjans (2014: 198-203) gezeigt hat, liegt doch eine gewisse Beziehung vor. Tatsächlich implizieren sowohl das Nicken wie auch traditionelle verbale Evidentheitsmarkierer (wie ja, natürlich, eben usw.), dass der Sprecher zum Zutreffen seiner Äußerung steht bzw. sie für wahr hält. Wahrheit und Evidentheit sind zwar unterschiedliche Nuancen, aber eine gewisse Ähnlichkeit liegt doch vor, insofern bei Evidentheit gerade das Zutreffen der Aussage als nicht zur Diskussion stehend markiert wird.

\footnotetext{
${ }^{3}$ Verweise auf Ausschnitte aus dem Datenkorpus entsprechen jeweils folgendem Muster: Äußerung [Videonummer]. [Nummer des Evidentheitsmarkierers im entsprechenden Video], [Anfangszeit des Ausschnittes][Ende des Ausschnittes]. Weitere Details zu den Materialen sowie eine Übersicht der Transkriptions- und Annotationskonventionen sind dem Anhang zu entnehmen.
}

ISSN 1615-3014 
Debras/Cienki (2012) gehen auf das Achselzucken und den sogenannten ,Tilt' ein. Für den Tilt - eine Geste, bei der der Kopf seitlich verkantet wird - gehen Debras und Cienki von einer breiten Skala von Funktionen aus, die von ,,affiliation“ über „,mitigated affiliation“ bis zur „disaffiliation“ reicht und auch noch die Verwendung als reines Hörersignal umfasst (Debras/Cienki 2012: 935). Sie präzisieren ihre Auffassung von ,,affiliation“ nicht, aber diese enthält zumindest eine bestätigende und mit Evidentheit zu verknüpfende Bedeutung. Das Achselzucken hingegen verknüpfen Debras/Cienki (2012: 936) explizit mit Selbstverständlichkeit und „logical or chronological necessity“4, obwohl sie auch andere mögliche Bedeutungen dieser Geste anführen.

Eine vierte Geste, die in der wissenschaftlichen Literatur schon öfter besprochen worden ist, allerdings mit unterschiedlichen Bezeichnungen, ist das intersubjektive (oder interaktive) Deiktikum. Sich auf u. a. Holler (2010) und Müller (1996) stützend umschreibt Schoonjans (2014: 208-209) die Funktionen dieser Geste als die Markierung von oder die Suche nach Einverständnis. Es handelt sich um eine Zeigegeste, die im Allgemeinen mit einer leicht gelockerten flachen Hand realisiert wird und mittels derer der Sprecher zeigt, dass er davon ausgeht, dass sich die Person, auf die die Geste gerichtet ist, mit der zugleich geäußerten Aussage einverstanden erklärt. Auch in diesem Fall lässt sich der Nuancenunterschied zwischen Einverständnis und Evidentheit überbrücken, insofern der Sprecher davon ausgeht, dass über das Evidente Einverständnis besteht.

Eine Geste, die Schoonjans (2014: 175-178) zufolge eine wesentlichere Beziehung zur Evidentheit aufweist, ist das Kopfschütteln: Diese Geste diene nicht nur der Verneinung, sondern sie könne zum Beispiel auch eine Aussage intensivieren oder ihr ausnahmsloses Zutreffen unterstreichen u.dgl. (vgl. dazu auch Kendon 2002 und McClave 2000). Die Kookkurrenz von Evidentheitsmarkierern und Kopfschütteln sei dann hauptsächlich auf die Ausnahmslosigkeitsbedeutung und die Intensivierungsfunktion, die sich auch in Evidentheitskontexten vorfinden lassen, zurückzuführen.

Weitere Gesten, die in Schoonjans' Daten (2014) häufiger mit verbalen Evidentheitsmarkierern kombiniert werden, sind die sogenannte ,Palm-lateral'-Geste und die sogenannte ,Horizontal-Palm'-Geste. Im ersteren Fall handelt es sich um eine Geste, bei der die geöffnete Hand (bzw. Hände) mit nach oben gerichteter Handfläche waagerecht zur Seite bewegt wird (bzw. werden). Bei der ,Horizontal-Palm'-Geste wird eine ähnliche Bewegung realisiert, allerdings sind diesmal die Handflächen nach unten gedreht. Im Anschluss an Kendons (2004) Analysen verknüpft Schoonjans (2014) beide Gesten mit Nuancen wie Evidentheit oder Unabänderlichkeit. Der ,Palm lateral' deute dann, Kendons (2004: 275) Qualifizierung dieser Geste als Zeichen von „,non-intervention“ entsprechend, auf das Zurückweisen jeglicher Verantwortung (,so ist es, ich kann es nicht ändern`), während ,horizontal Palm‘ alle Alternativen ausschließe und den Sachverhalt als nicht weiter zur Diskussion stehend darstelle (siehe u. a. auch Müller [2014: 145] und Bressem/Müller [2014: 1582]). Auch Calbris (2011: 179-180) vertieft sich in die Horizontal-Palm-Geste und erkennt ihr Bedeutungsnuancen wie Determinismus, Verpflichtung und Sicherheit zu.

\footnotetext{
${ }^{4}$ Wie dieser Begriff zu interpretieren ist, erläutern Debras und Cienki jedoch nicht.
} ISSN 1615-3014 
Beats (oder Taktstockgesten) können bei der Markierung von Evidentheit ebenfalls eine Rolle spielen. Beatgesten beschreiben eine (meist senkrechte) Hin-und-her-Bewegung, die dem Sprechrhythmus folgt, weisen aber formal (z. B. von der Handform her) eine große Vielfalt auf, was u. a. daran liegt, dass sie leicht mit anderen Gesten kombiniert werden können (vgl. u. a. Schoonjans 2014: 102). Die Basisfunktion des Beats ist die Betonung des zugleich verbal oder gestisch Geäußerten. So können sie anderen (verbalen oder gestischen) Evidentheitsmarkierern extra Nachdruck verleihen, aber auch bereits die Hervorhebung einer Aussage an sich kann als rhetorische Strategie dienen, um sie als evident oder nicht zur Diskussion stehend darzustellen.

Zum Schluss wurde in der Literatur auch auf das Heben der Augenbrauen in Evidentheitskontexten hingewiesen (siehe u. a. Jehoul 2014). Allerdings handelt es sich hier bislang nur um erste Beobachtungen; eine systematischere Analyse steht noch aus.

\section{Forschungsfrage, Material und Methode}

Wie aus der vorangehenden Übersicht deutlich geworden sein dürfte, sind die Studien, die auf die Verwendung bestimmter Gesten in Evidentheitskontexten hinweisen, recht zahlreich. Allerdings ist die Analyse von Gestik in Evidentheitskontexten im Allgemeinen nicht das eigentliche Thema dieser Arbeiten, sodass auch keine systematische Übersicht der mit Evidentheit zu verknüpfenden Gesten geliefert wird.5 Außerdem besprechen nicht all diese Arbeiten die Situation im Deutschen; es bleibt somit noch zu klären, inwiefern sich alle im vorigen Abschnitt gemachten Beobachtungen auch für das Deutsche geltend machen lassen. Ziel des vorliegenden Beitrags ist es, diese Forschungslücke zu füllen: Durch eine Analyse von Videodaten soll versucht werden, zu einem Gesamtbild der evidentheitsliierten Gesten im Deutschen zu kommen. Dabei geht es nicht nur darum, die Gesten an sich zu beschreiben: Auch deren Interaktion mit verbalen Evidentheitsmarkierern soll näher betrachtet werden.

Die nachfolgende Untersuchung stützt sich auf Daten aus fünf Fernsehgesprächen ${ }^{6}$, deren Gesamtdauer ca. 3 Stunden 15 Minuten beträgt. Diese Fernsehgespräche haben eines gemeinsam: Es handelt sich jeweils um eine Debatte, bei der die Teilnehmer(innen) versuchen, die Zuhörer (Opponenten wie Publikum) zu überreden. Dabei wird die Evidentheit häufig als rhetorisches Mittel zur Überzeugung eingesetzt.

Die räumliche Ausstattung der Fernsehgespräche ist immer so gestaltet, dass die Teilnehmer(innen) in ihrer Bewegungsfreiheit möglichst wenig gehindert werden und auf natürliche Weise am Gespräch teilnehmen können. Andererseits könnten sich die Beteiligten aber auch der Tatsache bewusst sein, dass sie an einem Gespräch teilnehmen, das im Fernsehen ausgestrahlt wird, was zugegebenermaßen seine Auswirkungen auf das jeweilige Sprecherverhalten (einschließlich der Gestik) haben könnte. Zudem ist darauf hinzuweisen, dass der Kamerastandpunkt nicht immer optimal ist und die Gesten der Sprecher folglich nicht immer deutlich zu erkennen sind. Diejenigen Fälle, in denen unklar ist, welche Geste realisiert wird bzw. ob überhaupt eine Geste realisiert wird, werden deshalb außer Betracht gelassen. Trotzdem führen diese Daten bereits zu interessanten Beobachtungen.

\footnotetext{
5 Eine nennenswerte Ausnahme stellt Jehouls (2014) Analyse zum Niederländischen dar.

${ }^{6}$ Genauere Angaben zu den einzelnen Videoaufnahmen sind dem Anhang zu entnehmen.
} 
Für die Zählung und Annotation der Gesten wurde mit den sogenannten ,stroke phrases` (Fricke 2012: 180) gearbeitet. Damit ist gemeint, dass die iterierte Realisierung einer Geste bei ein und derselben Äußerung jeweils als ein einziger Beleg gezählt wurde. Wenn also behauptet wird, dass eine bestimmte Geste vorliegt oder in Kombination mit einem anderen Evidentheitsmarkierer belegt ist, so kann es sich um eine einfache Realisierung der Geste handeln oder aber um eine iterierte Realisierung (d. h. mehrfach direkt aufeinander folgend). Zudem ist zu beachten, dass von einer Kombination zweier Gesten die Rede ist, wenn sie gemeinsam zu ein und derselben Äußerung realisiert werden. Impliziert ist nicht, dass die Gesten wirklich zeitgleich realisiert werden; auf die Reihenfolge kombinierter Gesten (wenn sie nicht zeitgleich realisiert werden) kann im Rahmen des vorliegenden Aufsatzes nicht weiter eingegangen werden.

Die Ergebnisse dieser Analyse werden im nachfolgenden Abschnitt erörtert. Dabei werden nacheinander die vorgefundenen verbalen (4.1) und gestischen Evidentheitsmarkierer (4.2) sowie deren Kookkurrenzverhalten (4.3) besprochen.

\section{$4 \quad$ Analyse der Daten}

\subsection{Verbale Evidentheitsmarkierer}

Der erste Schritt der Analyse fokussiert die im Datenmaterial enthaltenen verbalen Evidentheitsmarkierer an sich. Tabelle 1 liefert eine Übersicht der in den Daten vorgefundenen verbalen Evidentheitsmarkierer.

\begin{tabular}{|lcc|}
\hline verbales Element & absolute Frequenz & relative Frequenz \\
\hline ja & 93 & $34,83 \%$ \\
\hline natürlich & 74 & $27,72 \%$ \\
\hline doch & 22 & $8,24 \%$ \\
\hline klar & 20 & $7,49 \%$ \\
\hline eben & 20 & $7,49 \%$ \\
\hline sicher & 8 & $3,00 \%$ \\
\hline einfach & 8 & $3,00 \%$ \\
\hline selbstverständlich & 4 & $1,50 \%$ \\
\hline logisch & 4 & $1,50 \%$ \\
\hline also & 3 & $1,12 \%$ \\
\hline zweifellos & 2 & $0,75 \%$ \\
\hline offenkundig & 2 & $0,75 \%$ \\
\hline eindeutig & 2 & $0,75 \%$ \\
\hline eh & 2 & $0,75 \%$ \\
\hline was & 1 & $0,37 \%$ \\
\hline
\end{tabular}

\footnotetext{
${ }^{7}$ Auch Varianten dieser Elemente - wie ganz sicher, völlig klar, logischerweise usw. - wurden in die Zählung aufgenommen.
} 


\begin{tabular}{|lcc|}
\hline offensichtlich & 1 & $0,37 \%$ \\
\hline bekanntlich & 1 & $0,37 \%$ \\
\hline Total & 267 & $100,00 \%$ \\
\hline
\end{tabular}

Tabelle 1: Die Frequenz der verbalen Evidentheitsmarkierer

Aus Tabelle 1 geht hervor, dass sich natürlich und ja mit ihrer hohen Frequenz scharf gegen die anderen verbalen Elemente bzw. Gruppen abheben. Die Frequenz der anderen verbalen Evidentheitsmarkierer kommt, im Vergleich zur Gesamtzahl der verbalen Evidentheitsmarkierer, nicht über die $10 \%$ hinaus. Zu beachten ist auch, dass sich die verbalen Evidentheitsmarkierer untereinander kombinieren lassen. Solche Kombinationen sind aber eher selten und werden deshalb nicht eingehender besprochen.

Dass die Tabelle mehrere Modalpartikeln enthält (ja, doch, einfach, eben), soll an sich nicht verwundern. Nicht nur wurde im Abschnitt 2.2 bereits darauf hingewiesen, dass Thurmair (1989) Partikeln wie eben und einfach eine Evidenzbedeutung zuschreibt; außerdem passt Evidenz recht gut zu einem allgemeinen Abtönungskonzept, wie er etwa von Waltereit (2006) vorgeschlagen wurde. Waltereit zufolge ist Abtönung eine Perspektivierung bzw. Modifizierung der Äußerung im Hinblick auf die zu erwartende Hörerreaktion. Zu solchen Zwecken können tatsächlich auch Markierer der Evidentheit eingesetzt werden, gerade in persuasiven Kontexten wie den im Rahmen dieses Beitrags analysierten Debatten: Dass eine Behauptung keiner weiteren Beweise oder Argumente bedarf, sorgt dafür, dass sie sich als überzeugendes Argument eignet, denn was auf der Hand liegt und keiner weiteren Argumentation bedarf, lässt sich auch vom Gesprächspartner nicht so leicht hinterfragen oder widersprechen.

Allerdings dürfte deutlich sein, dass nicht alle verbalen Evidentheitsmarkierer gleich typische Beispiele der Kategorie ,Abtönung' sind wie die Modalpartikeln. Im Folgenden wird etwas ausführlicher auf die häufigsten verbalen Markierer eingegangen.

\subsubsection{Ja}

Mit insgesamt 93 Belegen $(34,83 \%)$ ist die Partikel ja der am meisten verwendete verbale Evidentheitsmarkierer. Die Kürze des Wortes dürfte eine partielle Erklärung für diese hohe Frequenz sein. Sprecher werden nämlich von einem ökonomischen Prinzip geführt und wollen während einer Rede möglichst Energie sparen.

Die hohe Frequenz wird daneben auch durch die Vielzahl von Funktionen von ja begründet ${ }^{8}$. Erstens kann ja in seiner Verwendung als Modalpartikel markieren, dass dem Sprecher zufolge der angesprochene Sachverhalt bekannt sein dürfte oder wenigstens als unstrittig gelten könne. Häufig handelt es sich zudem um eine Begründung von vorher Erwähntem oder gerade Geschehenem (ausführlicher dazu u. a. Bublitz 1978: 95-99; Thurmair 1989: 104-109 und Rinas 2007). In dieser Funktion wird ja auch im nächsten Datenausschnitt angewandt. Der Ausschnitt stammt aus der Fernsehdebatte zwischen Norbert Lammert, Sarah Jermutus, Pawel Hörnle und Nathanael Liminski. Die vier Teilnehmer diskutieren zu diesem Zeitpunkt über das Verhältnis zwischen dem akademischen Studium und der Beschäftigungsgarantie. Die

\footnotetext{
${ }^{8}$ Die Funktionen, die ja in einer Äußerung einnehmen kann, gehen weit über die hier angesprochenen, mit Evidentheit zu verknüpfenden Verwendungen hinaus. Andere Verwendungen von ja, zum Beispiel als Antwortpartikel, wurden bei der Analyse nicht berücksichtigt.
} 
zentrale Frage ist, inwiefern die Politik die Verteilung der Studienplätze steuern sollte und ob die Stärkung der Hochschulautonomie eine positive Entwicklung ist. Herr Lammert weist anhand der Partikel ja darauf hin, dass das Vorhandensein des betreffenden Trends (die Hochschulautonomie zu stärken) in den meisten Bundesländern den Gesprächspartnern und den Zuschauern bekannt ist bzw. dass sie selbst diesen Trend in der Gesellschaft haben beobachten können.

\section{2. Äußerung 1.63, 00:33:20.846-00:33:24.261}

Prof. Dr. Norbert Lammert: Den Trend (.) - [ den + wir ja ] - in den allermeisten + Bundesländern beobachten $()+$. können $+{ }^{\circ} \mathrm{hh}$ die Hochschulautonomie zu stärken

Allerdings kann die Evidentheit, die durch ja vermittelt wird, nicht immer als eine absolute Evidentheit gesehen werden: Die Aussage ist für den Sprecher evident, dürfte aber für den Gesprächspartner weniger bis gar nicht evident sein, wie im nachstehenden Beispiel. Je nach Kontext wird die Einstufung des Sachverhalts als evident dem Gesprächspartner , auferlegt', oder es ist die Frage impliziert, inwiefern der Gesprächspartner selber mit dieser Einstufung als evident einverstanden ist. Diese Verwendung von ja weist beachtliche Übereinstimmungen zum Vergewisserungssignal ja auf (vgl. Beispiel 1), da dies ebenfalls eine Bitte um die Bestätigung der Evidentheit darstellen kann (Bublitz 1978: 97-98; Schoonjans 2014: 67-71; Thurmair 1989: 104-109).

Das nachstehende Beispiel entstammt dem Anfang der Bambi-Debatte. Bushido weist hier auf die Diversität seines Publikums hin. So kommen zum Beispiel auch Rechtsradikale zu seinen Konzerten. Diese Rechtsradikalen trugen deutliche Symbole. „Schlimmer“ seien jedoch diejenigen, die keine solchen Symbole tragen und deren soziopolitische Einstellung dadurch nicht so klar zu erkennen ist. Für Bushido selber ist diese Aussage evident, er berücksichtigt aber die Möglichkeit, dass die Aussage dem Gesprächspartner nicht evident sein dürfte: Vielleicht sind Gesprächspartner anderer Meinung bzw. haben noch gar nicht an die Möglichkeit gedacht, dass diejenigen, die man nicht sofort erkennt, eine größere Gefahr sind als die anderen. Trotzdem markiert Bushido die Aussage als evident und unkontrovers, weil sie es seiner Ansicht nach ist.

\section{3. Äußerung 2.2,00:01:18.023-00:01:22.270}

Bushido: Die die Schlimmeren sind ja die die + man nicht am (2) nicht sofort erkennt $(-)$ genau +

Zudem lässt sich ja, wie oben bereits erwähnt wurde, als Vergewisserungssignal vorfinden. Da ja als Vergewisserungssignal der Aussage aber nicht immer eine evidente Wirkung verleiht, wird es hier nicht als reiner verbaler Evidentheitsmarkierer betrachtet (siehe 2.2).

Letztens kann ja als betonte Modalpartikel, synonymisch zu unbedingt oder auf jeden Fall (bzw. mit Negation auf keinen Fall), eine Aufforderung an den Gesprächspartner sein bzw. die bereits von einem anderen Element hervorgerufene Aufforderung verstärken, in Sätzen wie erzähl das ja nicht weiter! (Beispiel aus Duden online, s. v. ja) (vgl. auch u. a. Bublitz 1978: 100-103 und Thurmair 1989: 109). Obwohl diese Verwendung von ja in Evidentheitskontexten denkbar ist, findet sie sich in unseren Daten nicht vor. 


\subsubsection{Natürlich}

Das Adverb natürlich ist mit 74 Belegen (27,72 \%) ebenfalls ein gängiger Evidentheitsmarkierer. Diese hohe Frequenz hängt mit der starken Wirkung dieses Elements zusammen. Als Synonym von selbstverständlich sorgt natürlich für eine sehr deutliche Evidentheit, da die Evidentheit bei natürlich als Tatsache dargestellt wird. Darüber hinaus kann natürlich ausdrücken, dass eine Ahnung sich erfüllt, dass eine Erwartung eintrifft, wie im Beispiel er kam natürlich wieder zu spät (Duden online, s. v. natürlich).

Der nächste Datenausschnitt illustriert die evidentheitsmarkierende Verwendung von natürlich. Der Eupener Bürgermeister Elmar Keutgen erklärt den Gesprächspartnern und Zuschauern die unterschiedlichen Etappen, die bei der Realisierung eines Bauprojektes in einer Stadt zu durchlaufen sind, darunter die Rücksprache mit der zuständigen (in diesem Fall also mit der Deutschsprachigen) Gemeinschaft. Diese Etappe wird von ihm als logisch und unvermeidbar dargestellt.

4. $\quad$ Äußerung 4.50, 00:22:53.686-00:22:57.626

Elmar Keutgen: - - + [ dann (-) muss man natürlich ] + zu (--) zu der Deutschsprachigen Gemeinschaft gehen

\subsubsection{Doch}

22 der 267 Evidentheitsbelege enthalten das verbale Element doch $(8,24 \%)$, das genauso wie ja eine Vielfalt an Bedeutungsnuancen vermittelt und eine Selbstverständlichkeitsinterpretation bewirken kann. In einem Satz wie das hast du doch gewusst verleiht doch in seiner Verwendung als Modalpartikel der Aussage einen gewissen Nachdruck, der auch in der Evidentheit enthalten ist (vgl. u. a. Bublitz 1978: 112 und Thurmair 1989: 112-114): Die Evidentheit bewirkt eine Hervorhebung des selbstverständlichen Charakters der Aussage. Ein ähnliches Verfahren trifft auf die eben angeführte evidente Aussage mit doch zu.

Forschern wie Franck (1980: 181) zufolge kann die Partikel doch auch als Gedächtnisstütze eingesetzt werden, indem der Hörer mit der doch-Äußerung an etwas Bekanntes erinnert wird (siehe auch u. a. Bublitz 1978: 107-109 und Thurmair 1989: 113). In Ausschnitt 5 redet Bürgermeister Elmar Keutgen von den Bedürfnissen und Wünschen der Jugendlichen und erinnert daran, wie er diesen abgeholfen hat, nämlich indem er einen ,schönen Raum“ zur Verfügung gestellt hat. Den Zuhörern dürfte dies bereits bekannt sein, und durch doch signalisiert Keutgen, dass es sich bei der Aussage um eine Gedächtnisstütze handelt, weil diese Information an dieser Stelle im Gespräch durchaus relevant ist.

5. Äußerung 4.89: 00:49:25.550-00:49:37.500

Elmar Keutgen: $*\{$ Die FREIe $*\}{ }^{\circ}$ hh die die NICHT organisierte Jugend öh sprich Jugendheim und so weiter wir haben ihnen doch einen ${ }^{\circ} \mathrm{hh}$ öh schönen Raum (.) in der Schulstraße ${ }^{\circ} \mathrm{hh}$ wo gerade was eben Frau Niessen sagte der Stadtpark daneben ist

Eine von Thurmair (1989: 114-115) ebenfalls erwähnte Verwendung von doch, die mit Evidentheit zu verknüpfen ist, ist die Einstimmungserwartung. Der Evidentheit der Aussage wegen wird eine einstimmende Reaktion vonseiten des Gesprächspartners erwartet, wie im nachfolgenden Beispiel. 
6. $\quad$ Äußerung 2.11: 00:09:59.839-00:10:11.294

Peter Maffay: Aber der entscheidende Satz (.) und $\wedge$ ! der (.) und der IST $\wedge$ gefallen ! ${ }^{\circ} \mathrm{hh} \wedge$ ist doch $\wedge(--)$ zu sagen $(-)^{\wedge}$ - was ich gemacht hab - war (2) FALSCH (---) was ich gemacht hab war ein Fehler $\wedge$

Anhand der Partikel doch will Peter Maffay sichergehen, dass die Aussage auch für seine Zuschauer und Gesprächspartner evident ist. Durch das Einsetzen der Partikel thematisiert Maffay die Möglichkeit, dass man anderer Meinung sein könnte, und gibt zugleich an, dass er trotzdem damit rechnet, dass die Zuhörer einverstanden sind.

\subsubsection{Klar und eben}

Mit jeweils 20 Belegen sind klar und eben in den Videodaten gleich häufig belegt. Das heißt, dass sowohl der Evidentheitsmarkierer klar (bzw. ganz/völlig klar) als auch eben in 7,49\% der evidenten Äußerungen vorzufinden sind.

In einer Aussage wie klar bin ich einverstanden vermittelt das Adverb klar Selbstverständlichkeit bzw. Sicherheit (Duden online, s. v. klar). Das Adjektiv klar ${ }^{9}$, wie im Satz das ist doch ganz klar, ist ebenfalls als Evidentheitsmarkierer im Korpus belegt, insoweit es den Sachverhalt als ,fest umrissen, eindeutig, für jedermann übersichtlich und verständlich“ ( $D u$ den online, s. v. klar) darstellt. Ausschnitt 7 veranschaulicht die genaue Wirkung von klar. Die Tatsache, dass die westliche Kultur selbst nicht immer den Bedingungen der westlichen Werte entspricht, steht laut Frau Schwan außer Frage. Der Islam ist eindeutig nicht die einzige Kultur, die mit Defiziten zu kämpfen hat. Die Selbstverständlichkeit ihrer These markiert Frau Schwan anhand des verbalen Evidentheitsmarkierers ganz klar, der durch ganz sogar einen ausgesprochenen Nachdruck bekommt.

7. $\quad$ Äußerung 5.6, 00:30:39.723-00:30:41.063

Gesine Schwan: und dass es KEINESwegs so ist dass WIR immer auf der Höhe der westlichen Werte leben $(.)^{\wedge}<$ das ${ }^{\wedge}+$ ist GANZ KLAR $+>$

Die Modalpartikel eben hingegen wird eher als Verstärkung einer Feststellung benutzt bzw. dient dazu, einen Sachverhalt als evident, unabänderlich oder einzigmöglich darzustellen (siehe u. a. Bublitz 1978: 81-82 und Thurmair 1989: 120-123), und ist in der Hinsicht ein Evidentheitsmarkierer im wahrsten Sinne des Wortes. Diese Anwendung von eben findet sich im nachstehenden Beispiel: Obwohl, so Herr Schmidt-Salomon, die islamische Kultur sehr viele wertvolle Eigenschaften besitze, stehe seiner Meinung nach außer Frage, dass ihr auch viele schlechte Eigenschaften zuzuschreiben seien. Die zweite Aussage widerspricht also teilweise die erste. Dieser Gegensatz ist evident für Herrn Schmidt-Salomon: Er nimmt an, dass seine Zuhörer leicht einsehen, dass der Islam nicht nur gute, sondern auch schlechte Eigenschaften hat.

\footnotetext{
9 Ähnlich wie $j a$ können klar und eben auch andere als die hier besprochen evidentheitsliierten Funktionen erfüllen.
}

ISSN 1615-3014 
8. Äußerung 5.41, 00:59:44.332-00:59:48.140

Michael Schmidt-Salomon: und in diesen [^ Schatzkammern $\left.{ }^{\wedge}\right]$ findet man (---) [ sehr viel $\wedge^{\wedge}$ vernünftiges $]^{\wedge}$ und + menschenfreundliches $+{ }^{\circ} \mathrm{h}$ man findet aber eben auch $[-+$ Menschenverachtendes $-+^{\circ}$ hh und $]\left[{ }^{\wedge}\right.$ Sinnloses ${ }^{\wedge}$ ]

Darüber hinaus könnte man auch der Verwendung von eben als Antwortpartikel eine evidentheitsmarkierende Wirkung zuschreiben. Diese Verwendung ist jedoch in den untersuchten Daten nicht belegt und wird dementsprechend nicht weiter besprochen.

\subsection{Gestische Evidentheitsmarkierer}

Der zweite Teil der Analyse interessiert sich für die gestischen Evidentheitsmarkierer. Wie aus der nachstehenden Tabelle herzuleiten ist, ist der Beat die im Selbstverständlichkeitskontext am häufigsten produzierte Geste $(23,34 \%)$. An zweiter Stelle steht das Nicken $(18,81 \%)$, anschließend das Kopfschütteln (13,91\%), dann der Conduit $(10,22 \%)$ und an fünfter Stelle kommt der Tilt (9,69\%). Diese fünf Gesten werden unten (4.2.1) eingehender besprochen.

\begin{tabular}{|lcccccccc|} 
Beat & $\mathbf{4 4 3}$ & $\mathbf{2 3 , 3 4} \%$ & $\mathbf{1 9 7}$ & $\mathbf{6 5 , 6 7} \%$ & $\mathbf{9 3}$ & $\mathbf{3 1 , 0 0} \%$ & $\mathbf{1 0}$ & $\mathbf{3 , 3 3} \%$ \\
\hline Nicken & 357 & $18,81 \%$ & 189 & $\mathbf{6 3 , 2 1} \%$ & 105 & $35,12 \%$ & 5 & $1,67 \%$ \\
\hline Kopfschütteln & 264 & $13,91 \%$ & 141 & $63,23 \%$ & 77 & $34,53 \%$ & 5 & $2,24 \%$ \\
\hline Conduit & 194 & $10,22 \%$ & 99 & $66,89 \%$ & 45 & $30,41 \%$ & 4 & $2,70 \%$ \\
\hline Tilt & 184 & $9,69 \%$ & 108 & $70,59 \%$ & 42 & $27,45 \%$ & 3 & $1,96 \%$ \\
\hline $\begin{array}{l}\text { Augenbrauen } \\
\text { heben }\end{array}$ & 159 & $8,38 \%$ & 107 & $79,26 \%$ & 28 & $20,74 \%$ & 0 & $0,00 \%$ \\
\hline Achselzucken & 113 & $5,95 \%$ & 58 & $63,74 \%$ & 33 & $36,26 \%$ & 0 & $0,00 \%$ \\
\hline deiktisch & 102 & $5,37 \%$ & 57 & $64,77 \%$ & 30 & $34,09 \%$ & 1 & $1,14 \%$ \\
\hline metaphorisch10 & 42 & $2,21 \%$ & 23 & $65,71 \%$ & 11 & $31,43 \%$ & 1 & $2,86 \%$ \\
\hline horizontal Palm & 21 & $1,11 \%$ & 15 & $93,75 \%$ & 1 & $6,25 \%$ & 0 & $0,00 \%$ \\
\hline Palm lateral & 19 & $1,00 \%$ & 10 & $66,67 \%$ & 5 & $33,33 \%$ & 0 & $0,00 \%$ \\
\hline Total & 1898 & $100,00 \%$ & 1004 & $66,80 \%$ & 470 & $31,27 \%$ & 29 & $1,93 \%$ \\
\hline
\end{tabular}

Tabelle 2: Die Frequenz der gestischen Evidentheitsmarkierer

Zwecks einer besseren Beobachtung des Zusammenwirkens von Gestischem und Verbalem ist unterschieden worden zwischen den Belegen einer Geste in Kombination mit anderen gestischen (dritte Spalte) oder verbalen Evidentheitsmarkierern (vierte Spalte) und den Belegen der bloßen Geste (fünfte Spalte). Allerdings wird den Kombinationen verbaler und gestischer Evidentheitsmarkierer, die wesentlich seltener sind als Verbindungen gestischer Evidentheitsmarkierer untereinander, ein separater Abschnitt (4.3) gewidmet. In Bezug auf die erwähnten Frequenzen der Kombinationen ist erneut darauf hinzuweisen, dass die kombinierten Evidentheitsmarkierer nicht unbedingt zeitgleich realisiert werden, sondern in einer Äußerung auch direkt aufeinander folgen können.

\footnotetext{
${ }^{10}$ Den Conduit-Gesten liegt zwar auch eine Metapher zugrunde (eben die Conduit-Metapher), aber trotzdem werden sie hier getrennt von den anderen metaphorischen Gesten besprochen, weil es sich um eine Metapher handelt, die in der Interaktion im Allgemeinen recht häufig (gestisch) realisiert wird und besonders in Evidentheitskontexten auffällig häufiger eingesetzt wird als andere Metaphern (vgl. dazu auch Abschnitt 4.2.1.4). Unter ,metaphorisch“ werden hier also nur die anderen metaphorischen Gesten verstanden.
} 
Was die bloßen gestischen Evidentheitsmarkierer betrifft, ist in der Tabelle 2 festzustellen, dass nur 1,93\% der gestischen Evidentheitsmarkierer von sich aus die Evidentheit einer Aussage gewährleisten. Das Fehlen einer eindeutigen Interpretation für die Mehrheit der Gesten dürfte hier eine Rolle spielen. Bestimmte Gesten können tatsächlich in unterschiedlichen Kontexten zu unterschiedlichen Zwecken eingesetzt werden. Die Interpretation der Aussage hängt dann von einer Fülle von Elementen ab, die zusammen die Bedeutung bilden.

Auffällig ist das häufigere einzelne Vorkommen eines Beats $(3,33 \%)$, denn die Vielfalt an Interpretationen ist bei dieser Geste gerade sehr groß (siehe Abschnitt 4.2.1.1). Die stark betonende Wirkung des Beats dürfte eine Erklärung für diese gesteigerte Frequenz liefern. Oben wurde schon auf die betonende Dimension der Evidentheit hingewiesen und die Hervorhebung, die der Beat bewirken kann, schließt an diese Eigenschaft der Selbstverständlichkeit an.

Schließlich ist noch zu bemerken, dass diejenigen Gesten, bei denen infolge des Kamerastandpunkts nicht festgestellt werden kann, ob noch andere Gesten vorliegen oder nicht, nur für die Berechnung der absoluten Gesamtfrequenz (zweite Spalte) berücksichtigt worden sind. Die Summe der Frequenzen in der dritten, vierten und fünften Spalte einerseits und die absolute Frequenz der zweiten Spalte andererseits sind demzufolge nicht immer gleich.

\subsubsection{Frequente gestische Evidentheitsmarkierer}

\subsubsection{Der Beat}

Die Einfalt, die die Bewegung eines Beats kennzeichnet, sorgt dafür, dass die Geste einfach anzuwenden ist, was die hohe Frequenz zum Teil begründen dürfte. Auch die Funktion könnte allerdings eine Rolle spielen: Der Beat kann zu verschiedenen Zwecken angewandt werden, vor allem aber dient er der Betonung, die in persuasiven Kontexten ein wichtiges rhetorisches Hilfsmittel darstellt.

Die Hervorhebung ist der wichtigste Generalnenner des Beats und der Evidentheit, denn anhand eines Evidentheitsmarkierers will der Sprecher unterstreichen, dass die Aussage evident ist. Trotzdem ist die Verbindung des Beats zur Selbstverständlichkeit nicht immer durchschaubar und gleich stark ausgeprägt, sondern eher vage und zweifelhaft. Die Grenze zwischen bloßer Evidentheit und einer evidentheitsnahen Interpretation ist für den Beat somit schwer zu fassen.

Im nachstehenden Beispiel wird der Beat mit der Partikel ja und dem Adverb natürlich kombiniert, was die Stärke der Selbstverständlichkeit steigert. Die Standbilder veranschaulichen die genaue Realisierung des betreffenden Beats. Es handelt sich in diesem Fall um einen Beat, der sowohl mit dem Kopf als auch mit der rechten Hand ausgeführt wird, wie das Superscript angibt.

Herr Schmidt-Salomon knüpft am Anfang seiner Intervention an die Rede des vorherigen Sprechers an: Er gibt zu erkennen, dass er in diesem Punkt mit ihm einverstanden ist, dass Muslime und Christen gleich behandelt werden müssen, und markiert diese Aussage als selbstverständlich. 
9. $\quad$ Äußerung 5.89, 00:31:39.431-00:31:45.574

Michael Schmidt-Salomon: ${ }^{\wedge}$ Kopf + rechte Hand wir haben ja die weltanschauliche Neutralität des Staates (.) und das beDEUTet (-) dass wir naTÜRlich Muslime gleich behandeln müssen mit Christen $\wedge$
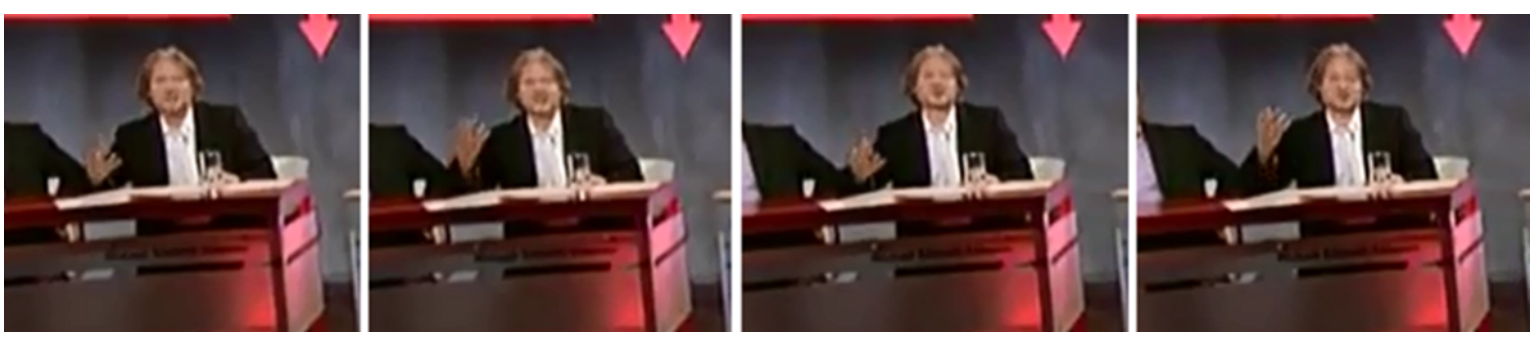

Abbildung 1: Handbeat zu Ausschnitt 9
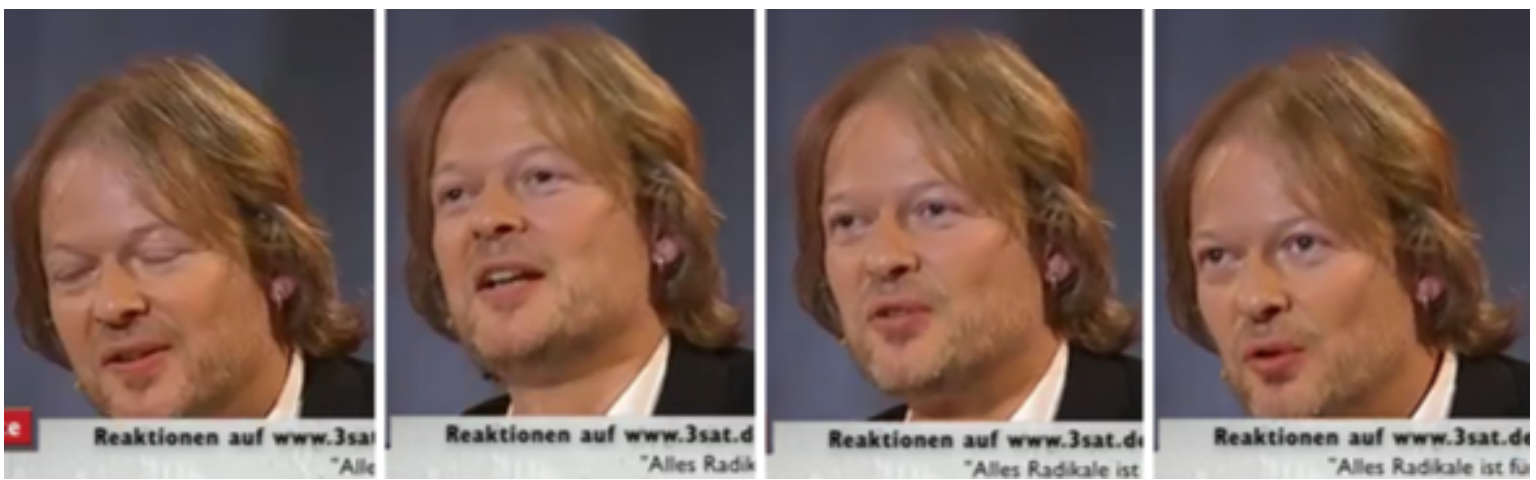

Abbildung 2: Kopfbeat zu Ausschnitt $9^{11}$

\subsubsection{Das Nicken}

Die Bedeutung des Nickens ähnelt jener des Beats, obwohl das Nicken vor allem die Bestätigung fokussiert (vgl. Schoonjans 2014: 201-202), die Betonung aber ebenfalls in den Vordergrund stellen kann. Die Schlussfolgerung lautet hier also wiederum, dass die Verbindung zwischen dem Nicken und der Evidentheit unleugbar ist, jedoch nicht immer klar und deutlich festzustellen ist. Die Interpretation nähert sich oft der Evidentheit, ohne diese wirklich auf den Punkt zu bringen. Allerdings deutet das Nicken nicht immer an, dass der Sprecher zu seiner Aussage steht bzw. sie für wahr oder gar bekannt hält: Darüber hinaus kann das Nicken auch eine Bitte um Bestätigung signalisieren. In dem Fall wendet sich der Sprecher an den Gesprächspartner mit der Frage, ob die Aussage für ihn ebenso evident ist. Das Nicken leistet insofern eine Bitte um eine ,projected affirmative answer“ (Whitehead 2011: 113).

Die bestätigende Funktion des Nickens offenbart sich in Beispiel 10, dass erneut der Eupener Wahldebatte entstammt. In diesem Ausschnitt kritisiert Herr Baumgarten die Weise, auf die der heutige Bürgermeister bei der Neugestaltung der Innenstadt vorgegangen ist. Anhand u. a. des Nickens bestätigt er (bzw. markiert als evident), dass die Innenstadt von den Bürgern benutzt wird und dass also die Perspektive der Bürger bei der Planung der Bauarbeiten eine zentrale Rolle zu spielen hat. Dieses Nicken wird darüber hinaus mit der Partikel ja, ebenfalls

\footnotetext{
${ }^{11}$ Wegen der wechselnden Kameraperspektive wird zur Verdeutlichung in Abbildung 1 der Anfang der Beatsequenz, in Abbildung 2 dagegen deren Ende gezeigt. Beide Beats verlaufen jedoch parallel während des gesamten im Ausschnitt 9 markierten Teils.
} 
einem bestätigenden Evidentheitsmarkierer, verknüpft. Für eine eingehendere Erörterung der Kombination von ja und Nicken, siehe Abschnitt 4.3.2.

10. Äußerung 4.5, 00:03:32.672-00:03:38.077

Werner Baumgarten: + man beginnt also mit $\operatorname{dem}^{\wedge}$ Benutzer $^{\wedge}($.$) man beginnt mit dem$ ${ }^{\circ}$ hh Bürger $+{ }^{\text {iteriert }}$ der [ irgendwann ja auch diese $+(-) /$ diese Innenstadt $\backslash$ benutzen muss]

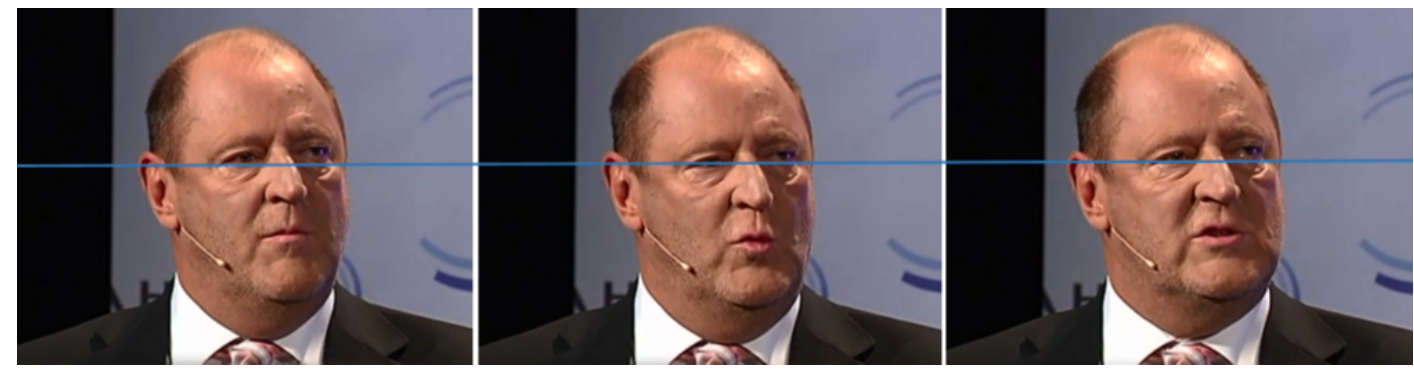

Abbildung 3: Nicken zu Ausschnitt 10 ${ }^{12}$

\subsubsection{Das Kopfschütteln}

Schoonjans (2014: 174-206) hat für das Deutsche bereits die Korrelation zwischen dem Kopfschütteln und der Evidentheit bzw. der evidentheitsmarkierenden Partikel einfach eingehend untersucht. In diesem Zusammengang verweist er auf die ,funktionale Übereinstimmung“ (Schoonjans 2014: 177) zwischen den beiden: Sowohl das Kopfschütteln als auch die Partikel einfach können zum Beispiel eine Intensivierung auslösen oder Ausnahmslosigkeit bzw. Einzigmöglichkeit markieren. Die höhere Frequenz des Kopfschüttelns in Evidentheitskontexten ist also nicht erstaunlich.

Das Kopfschütteln wird jedoch nicht ausschließlich mit einfach verbunden, wie es auch Ausschnitt 11 zeigt. Es kann beispielsweise auch mit der Partikel eben kombiniert werden. Das Kopfschütteln behält aber seine Funktion als Markierer von Einzigmöglichkeit oder Ausnahmslosigkeit. Die Stadt Eupen habe, so Herr Keutgen, einfach eine Erneuerung gebraucht; er habe als Bürgermeister also keine andere Wahl gehabt als eine solche durchzuführen. Dieses Beispiel verdeutlicht also, wie das Kopfschütteln die Einzigmöglichkeit ausdrücken kann.

11. Äußerung 4.1,00:01:46.672-00:01:51.010

Elmar Keutgen: $\left\{\right.$ aber * ich glaube - $^{\text {iteriert }}{ }^{\circ} \mathrm{hh} \mathrm{-}$ \} öh (--) die Situation war eben so * dass der Zeitpunkt gekommen war

\footnotetext{
12 Da das Nicken in diesem Fall ziemlich subtil ist, wurde im Hinblick auf die Erkennbarkeit der Bewegung eine Achslinie hinzugefügt (ähnlich auch u. a. Abbildung 4).
}

ISSN 1615-3014 


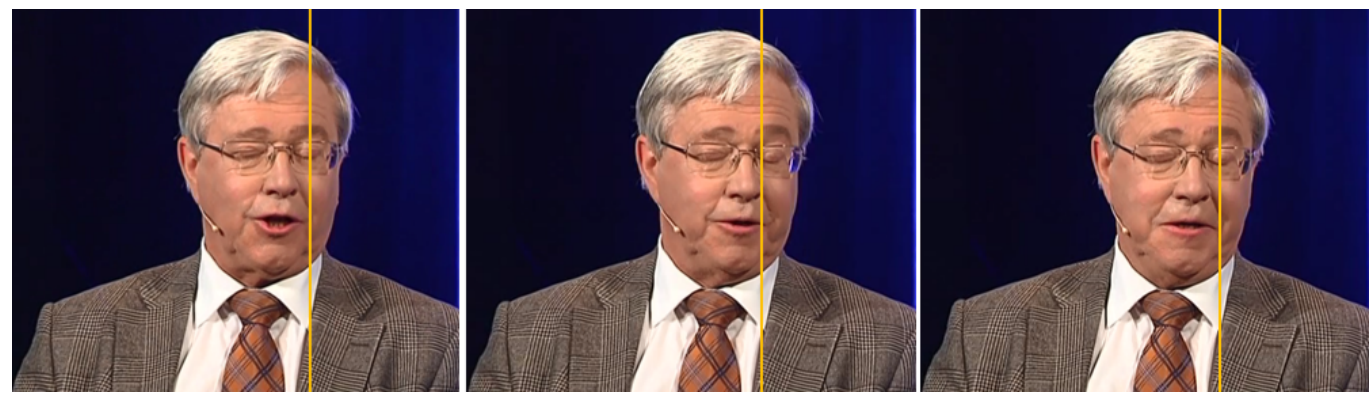

Abbildung 4: Kopfschütteln zu Ausschnitt 11

\subsubsection{Der Conduit}

Unter dem Nenner ,Conduit` werden an dieser Stelle mehrere Gesten zusammengeführt, die alle auf dieselbe Metapher zurückgehen, die sogenannte ,Conduit'-Metapher, nach der sprachliche Interaktion als ein Austausch von Objekten (die Äußerungen) repräsentiert wird (McNeill 2005: 45 und Müller 2004: 238). Der Informationstransfer in einer Assertion kann zum Beispiel als das Anbieten des Gesagten auf der flachen Hand (,bietender Conduit') oder als das Hinstellen des Gesagten in den Gestenraum (,hinstellender Conduit') dargestellt werden.

Die Conduit-Gesten können im Diskurs zu unterschiedlichen Zwecken und mit unterschiedlichen Nuancen eingesetzt werden (vgl. u. a. Kendon 2004: 266-270 und Müller 2004 für eine ausführlichere Übersicht typischer Verwendungskontexte des ,bietenden Conduits", den sie als ,Palm Presenting ' bzw. ,Palm Up Open Hand‘ bezeichnen). Aus den Videodaten geht hervor, dass die Conduit-Gesten auch in Evidentheitskontexten eine nicht zu vernachlässigende Rolle spielen. Dies dürfte auf den Umstand zurückzuführen sein, dass Conduits häufig eine intersubjektiv-deiktische Komponente (vgl. 2.3) enthalten und somit oft auf Bekanntes, Unkontroverses oder Evidentes hinweisen (siehe zur Beziehung von bietendem Conduit und Evidentheit auch u. a. Müller 2010: 56).

Der nachfolgende Ausschnitt führt Herrn Keutgen während der Realisierung eines ,bietenden“ Conduits auf. Dieser Conduit wird mit den beiden Händen ausgeführt und bietet dem Gesprächspartner die evidente Aussage an. Bevor Herr Keutgen mit seiner Aussage anfängt, hält er die beiden Hände gefaltet. Am Anfang des Satzes öffnet er die Hände, wie in Abbildung 5 zu sehen ist, und bildet so eine Art von Tableau, auf dem er seinem Gesprächspartner die Aussage und deren Evidentheit eigentlich anbietet (Müller 2010: 56). Ob dieser Conduit eine intersubjektiv-deiktische Komponente enthält, kann jedoch nicht eindeutig bestätigt werden, da aufgrund der Kameraperspektive die genaue Orientierung der Geste nur schwer festzulegen ist bzw. nicht bestimmt werden kann, ob sie tatsächlich auf die Zuhörer gerichtet ist.

12. Äußerung 4.28, 00:22:53.990-00:22:57.523

Elmar Keutgen: - - + [ ${ }^{2}$ Hände bietend dann (-) muss man natürlich ] + zu (--) zu der deutschsprachigen Gemeinschaft gehen 


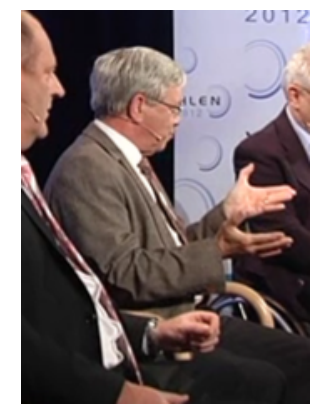

Abbildung 5: Bietender Conduit zu Ausschnitt 12

\subsubsection{Der Tilt}

In der Arbeit von Debras/Cienki (2012) fungiert der Tilt nicht als (reiner) Evidentheitsmarkierer. Im hier analysierten Korpus scheint er dennoch eine in Evidentheitskontexten relativ wichtige Geste zu sein. Diese Feststellung dürfte auf die Möglichkeit zurückzuführen sein, durch einen Tilt „affiliation“ (Debras/Cienki 2012: 935) zu markieren. Obwohl Debras und Cienki ihre Interpretation von ,affiliation“ nicht weiter verdeutlichen, schwingt hier durchaus eine Bestätigung mit, und diese bestätigende Interpretation ist ebenfalls in der Evidentheit enthalten. Diese bestätigende Wirkung des Tilts ist im nächsten Datenausschnitt zu erkennen.

Der Ausschnitt entstammt der politischen Debatte mit Bundestagspräsident Lammert. Zu diesem Zeitpunkt handelt die Diskussion von der Frage, welchen Einfluss die Eltern heute noch haben, vor allem im politischen Bereich. Herr Lammert ist der Meinung, dass die Neigung zu (bzw. die Abneigung von) politischem Engagement bei Jugendlichen von den Eltern mitbeeinflusst werde und nicht einfach mittels politischen Maßnahmen umgestellt werden könne. Diese These wurde vorher auch von den anderen Gesprächsteilnehmern geäußert, und Herr Lammert scheint hier einen Tilt einzusetzen, um dieser These zuzustimmen.

13. Äußerung 1.17, 00:26:59.749-00:27:13.854

Prof. Dr. Norbert Lammert: $\{$ links $* /$ deswegen $\backslash *\}$ ist es auch + sicher $+\wedge$ illusorisch $\wedge$ (3) wenn die Politik (---) diese Bedeutung (.) des Elternhauses durch welche Art von staatlichen Maßnahmen auch immer $()>$.+ kompenSIEren wollte $+<$

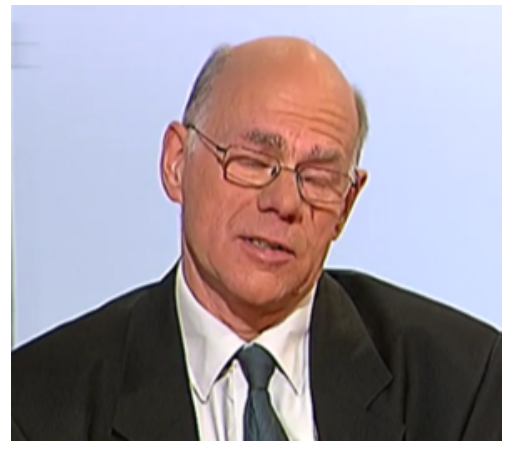

Abbildung 6: Tilt zu Ausschnitt 13 


\subsubsection{Kombinationen gestischer Evidentheitsmarkierer}

Bei der Besprechung von Tabelle 2 (4.2) wurde bereits darauf hingewiesen, dass gestische Evidentheitsmarkierer häufig untereinander kombiniert werden. Dies gilt insbesondere für den horizontal Palm $(93,75 \%)^{13}$, das Heben der Augenbrauen $(79,26 \%)$ und den Tilt $(70,59 \%)$, die meist mit einer anderen Geste gemeinsam eingesetzt werden. Allerdings weisen diese Gesten ein breites Kombinationsspektrum auf, sodass die Kombinationen mit diesen Gesten (auch aufgrund der relativen Seltenheit dieser Gesten an sich) trotzdem nicht zu den häufigsten Kombinationen in den Daten gehören.

Tabelle 3 listet die häufigsten Kombinationen gestischer Evidentheitsmarkierer auf. In der Tabelle wird jeweils angegeben, wie viele Belege der Geste in der linken Spalte mit einem oder mehreren Belegen der Geste in der ersten Zeile kombiniert werden. Dass in einer Äußerung mehrere Beatgesten und ein einziges Nicken vorkommen können, erklärt also zum Beispiel, wieso die absolute Frequenz für Nicken+Beat und Beat+Nicken nicht gleich ist.

\begin{tabular}{|l|c|c|c|c|c|c|}
\hline & \multicolumn{2}{|c|}{ Kopfschütteln } & \multicolumn{2}{c|}{ Nicken } & \multicolumn{2}{c|}{ Beat } \\
\hline Kopfschütteln & & & 80 & $30,30 \%$ & 78 & $29,55 \%$ \\
\hline Nicken & 83 & $23,25 \%$ & & & 150 & $42,02 \%$ \\
\hline Beat & 80 & $18,06 \%$ & 148 & $33,41 \%$ & & \\
\hline
\end{tabular}

Tabelle 3: Die häufigsten Kombinationen zwischen Gesten

Die nennenswerten Verbindungen beschränken sich auf die Kombination von einem Beat und einem Nicken sowie die Kombination von einem Nicken und einem Kopfschütteln. Die Verknüpfung von einem Nicken und einem Beat ist sowohl aus der Perspektive des Nickens $(42,02 \%)$ als auch aus der Perspektive des Beats $(33,41 \%)$ eher frequent. Diese Kombination sieht dann beispielsweise wie im Ausschnitt 14 aus. Die Evidentheit der Aussage wird erstens anhand eines iterierten Nickens hervorgehoben, und diesem Nicken folgt ein einfacher evidentheitsmarkierender Beat.

Das Nicken von Herrn Schmidt-Salomon hat hier die gleiche Funktion wie in Beispiel 10 (4.2.1.2.): Es bestätigt die Evidentheit der Aussage. Für Herrn Schmidt-Salomon ist es selbstverständlich, dass die humanistischen Werte nicht nur dem Westen gehören. Mittels des Nickens bestätigt er, dass diese Aussage für seine Zuhörer evident sein dürfte. Diese bestätigende Funktion des Nickens wird von dem Beat übernommen. Mit dem Kopfbeat betont Herr Schmidt-Salomon darüber hinaus die Aussage und deren Selbstverständlichkeit.

14. Äußerung 5.4(Nicken)/5.3(Beat), 00:06:31.153-00:06:39.499

Michael Schmidt-Salomon: denn (-) humanistische Werte $(-)+{ }^{\text {iteriert }}$ sind keine exklusiven Besitztümer des $+\wedge^{\wedge \text { Kopf einfach }}$ WESTens ${ }^{\wedge}{ }^{\circ} \mathrm{hh}$ es handelt sich vielmehr $(-)$ um ein Weltkulturerbe der Menschheit

\footnotetext{
${ }^{13}$ Hier ist jedoch Vorsicht geboten, denn der horizontal Palm ist im Korpus nicht sehr häufig belegt (siehe Tabelle 2).
} 


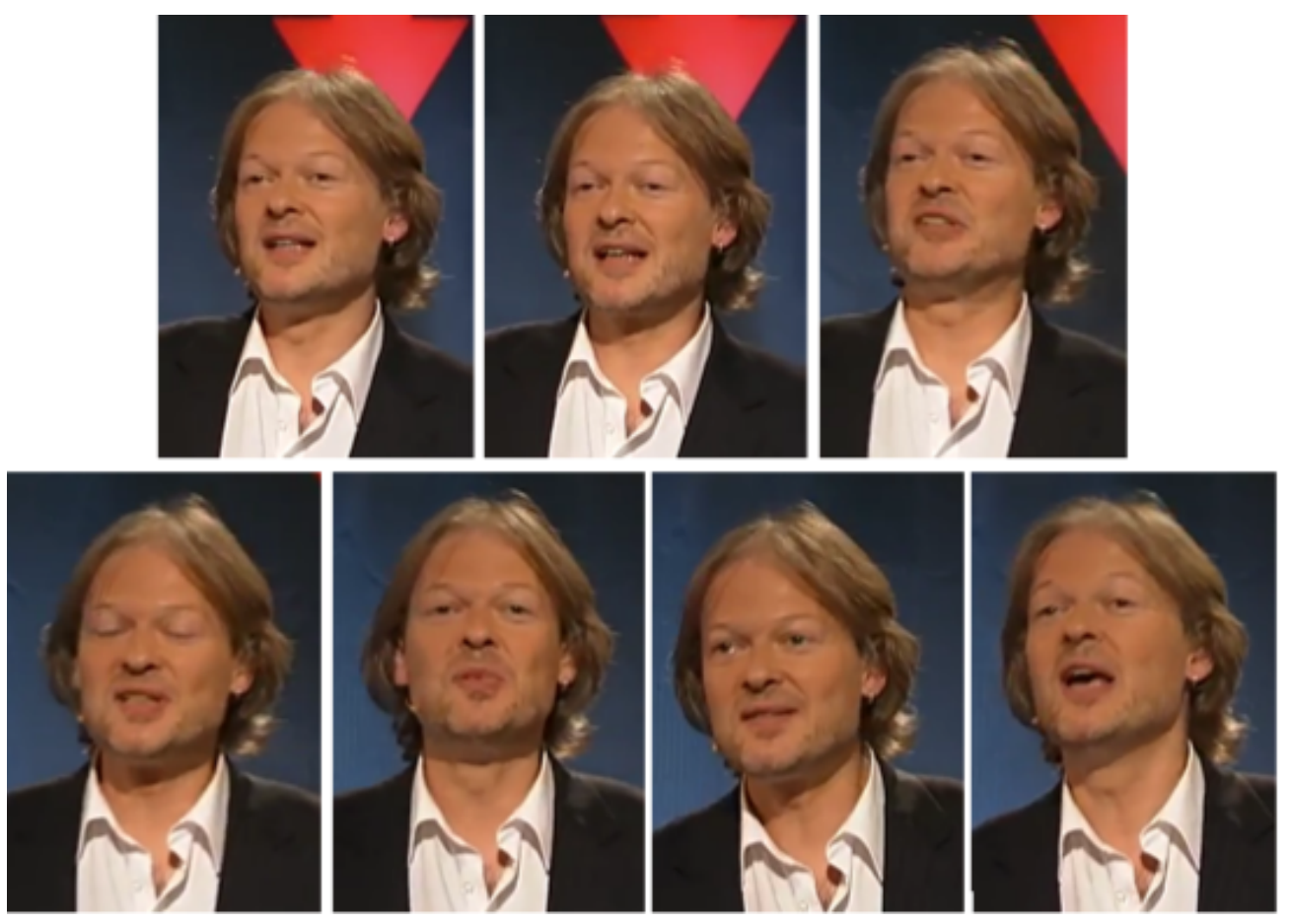

Abbildung 7: Nicken gefolgt von einem Kopfbeat zu Ausschnitt 14

Die Verbindung von einem Beat und einem Nicken kann freilich auch als eine Einbettung realisiert werden. Ein oder mehrere kurze Beats sind dann in einer längeren Nicksequenz integriert oder umgekehrt.

Auch die Kombination von Nicken und Kopfschütteln taucht regelmäßig in den Videodaten auf. Für das Nicken liegt hier eine relative Frequenz von 23,25\% vor; für das Kopfschütteln handelt es sich um 30,30\% der Belege. In den beiden Fällen folgt eine der beiden Gesten unmittelbar auf die andere.

\subsection{Verbale und gestische Evidentheitsmarkierer}

Das Hauptinteresse dieses Aufsatzes liegt jedoch bei der Kookkurrenz verbaler und gestischer Einheiten in Evidentheitskontexten. Diese Kookkurrenz kann aus zwei Perspektiven betrachtet werden: der verbalen und der gestischen. So kann einerseits aus der Tabelle 4 abgelesen werden, dass 55,00 \% der Instanziierungen von klar mit einem Kopfschütteln einhergehen. Dieser hohe Prozentsatz könnte den Eindruck wecken, dass hier eine gewisse Korrelation zwischen klar und dem Kopfschütteln vorliegt. Allerdings lässt sich dies aus der Perspektive der Geste nicht aufrechterhalten, denn es handelt sich nur um 4,17\% der Instanziierungen eines Kopfschüttelns (11 von 264). Es scheint also nur in einer Richtung eine deutliche Tendenz vorzuliegen, was mit der niedrigeren Frequenz von klar zusammenhangen dürfte: Klar zeigt einen starken Hang zum Kopfschütteln (11 von 22 Belegen), macht aber nur einen kleinen Anteil der Belege des an sich viel frequenteren Kopfschüttelns aus. Dies zeigt also, dass man, um ein richtiges Bild der Kookkurrenztendenzen zu bekommen, jeweils die beiden Perspektiven in Betracht ziehen sollte.

Die auffälligsten Kombinationen aus der Tabelle 4 werden unten eingehender besprochen. Dabei stellt die Tabelle bereits eine an die Frequenz orientierte Auswahl dar. Sie bietet eine Übersicht der frequentesten Kombinationen, aus verbaler wie aus gestischer Sicht. 
Weiterhin ist noch anzumerken, dass eine Kombination mit einem Evidentheitsmarkierer heißt, dass dieser Evidentheitsmarkierer ein oder mehrere Male in dieser Kombination belegt ist. Ein Evidentheitsmarkierer wird also mit einer oder mehreren Instanziierungen eines anderen Evidentheitsmarkierers verknüpft. Wenn zum Beispiel zu einer Äußerungseinheit mit natürlich zweimal der Kopf ,getiltet` wird, gilt das also als nur ein einziger Kombinationsbeleg aus der Sicht der verbalen Partikel (allerdings als zwei Belege aus der Perspektive der Geste, denn es sind zwei Instanziierungen der Geste betroffen).

\begin{tabular}{|c|c|c|c|}
\hline & $\begin{array}{c}\text { Perspektive } \\
\text { der Geste }\end{array}$ & $\begin{array}{c}\text { Perspektive } \\
\text { des Verbalen }\end{array}$ & \\
\hline Kopfschütteln & $7,95 \%$ & $28,38 \%$ & natürlich \\
\hline Nicken & $8,96 \%$ & $43,24 \%$ & natürlich \\
\hline Achselzucken & $9,73 \%$ & $14,86 \%$ & natürlich \\
\hline Tilt & $8,70 \%$ & $21,62 \%$ & natürlich \\
\hline $\begin{array}{r}\begin{array}{r}\text { Augenbrauen } \\
\text { heben }\end{array}\end{array}$ & $8,81 \%$ & $18,92 \%$ & natürlich \\
\hline $\begin{array}{r}\text { Intersubjektives } \\
\text { Deiktikum }\end{array}$ & $12,77 \%$ & $8,11 \%$ & natürlich \\
\hline Beat & $5,64 \%$ & $33,78 \%$ & natürlich \\
\hline Kopfschütteln & $4,17 \%$ & $55,00 \%$ & klar \\
\hline Kopfschütteln & $2,27 \%$ & $75,00 \%$ & einfach \\
\hline Nicken & $2,80 \%$ & $50,00 \%$ & eben \\
\hline Beat & $2,48 \%$ & $55,00 \%$ & eben \\
\hline Kopfschütteln & $3,03 \%$ & $36,36 \%$ & doch \\
\hline Nicken & $11,76 \%$ & $45,16 \%$ & ja \\
\hline Beat & $5,87 \%$ & $27,96 \%$ & ja \\
\hline Kopfschüitteln & $8,33 \%$ & $23,66 \%$ & ja \\
\hline
\end{tabular}

Tabelle 4: Übersicht der häufigsten Kombinationen von gestischen und verbalen Evidentheitsmarkierern

\subsubsection{Nicken und natürlich}

Auf die Verknüpfung zwischen natürlich bzw. dem Nicken und der Evidentheitsbedeutung wurde oben (Abschnitt 4.1.2 für natürlich, Abschnitt 5.2.1.2 für das Nicken) bereits eingegangen. Was natürlich und Nicken aber gemeinsam haben, ist die Eigenschaft, etwas betonen bzw. intensivieren zu können. Außerdem ergänzen sie sich relativ gut, insofern natürlich ein sehr starker Evidentheitsmarkierer ist, das Nicken hingegen nicht ausschließlich mit Selbstverständlichkeit zu verknüpfen ist, sondern ein breiteres Verwendungsspektrum aufweist.

Jedenfalls ist die Kombination von natürlich und Nicken aus beiden Perspektiven vergleichsweise frequent: In 8,96\% der Instanziierungen eines Nickens ist das verbale Element natürlich vorzufinden und 43,24\% der Instanziierungen von natürlich gehen mit einem Nicken einher. Dass die Anzahl der Instanziierungen von natürlich bei einem Nicken nicht so hoch ausfällt wie in umgekehrter Richtung, muss nicht unbedingt problematisch sein, denn 8,96\% von 264 Belegen stellt immer noch eine beachtliche Zahl dar. Beispiel 13 veranschaulicht, wie diese beiden Evidentheitsmarkierer sich kombinieren lassen. Elmar Keutgen ist noch immer dabei, sich gegen die Vorwürfe in Bezug auf die Planung der großen Erneuerung der Eu- 
pener Stadtmitte zu wehren. Die Oppositionsparteien behaupten, es wäre besser gewesen, anders an das Projekt heranzugehen und sich so Zeit zu sparen. Keutgen gesteht, mittels der Kombination von einem Nicken und natürlich, dass er das hätte machen können und dass die Bauarbeiten dann schneller abgelaufen wären, qualitativ gesehen hätte es aber keine Vorteile gebracht. Das Nicken und natürlich vermitteln zusammen eine bestätigende und selbstverständliche Botschaft.

15. Äußerung 4.11 (Nicken)/4.18 (natürlich), 00:06:38.907-00:06:41.923

Elmar Keutgen: $+{ }^{\text {iteriert }}$ da wären wir natürlich (.) RASCHer fertig geworden $\boldsymbol{+}$ als heute

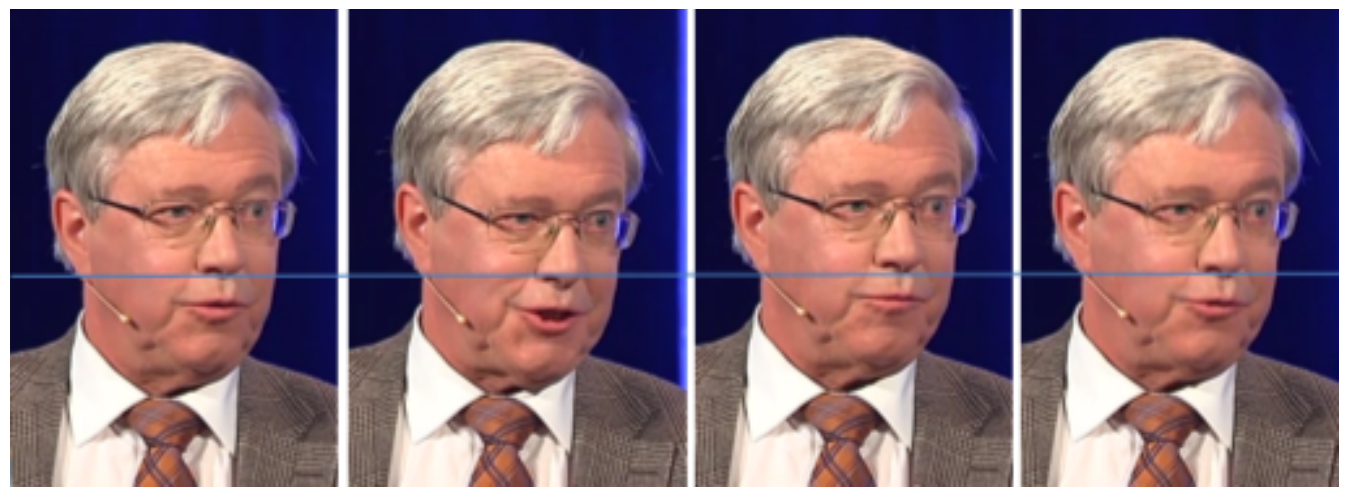

Abbildung 8: Nicken mit natürlich (zu Ausschnitt 15)

\subsubsection{Nicken und $j a$}

Aufgrund der Häufigkeit der Verbindung von einem ja und einem Nicken (45,16\% aus der Perspektive von $j a$ und $11,76 \%$ aus der Perspektive des Nickens) lohnt es sich, auch diese Kombination eingehender zu besprechen.

Die Funktionen von ja und dem Nicken im Bereich der Evidentheit sind oben schon angesprochen worden: Ja kann, genauso wie das Nicken, eine Bitte um die Bestätigung der Evidentheit darstellen (siehe Abschnitt 4.1.1 für ja und Abschnitt 4.2.1.2 für das Nicken). Des Weiteren eignen sich die beiden Evidentheitsmarkierer als Ausdruck der Bestätigung. Ihre Interpretation könnte in diesem Fall paraphrasiert werden als ,wie Sie (ja) wissen“. Das Nicken bzw. das ja bestätigt somit, dass der Sachverhalt dem Gesprächspartner bereits bekannt und sogar evident sein sollte. Diese bestätigende Wirkung der Kombination der beiden Evidentheitsmarkierer spielt im untenstehenden Beispiel deutlich eine Rolle. Hier bestätigt Herr Schmidt-Salomon, dass er schon darauf hingewiesen hat, dass die westlichen Werte ursprünglich eher als östliche Werte zu betrachten waren, und gibt so zu erkennen, dass er davon ausgeht, dass dies den Zuschauern bekannt ist.

16. Äußerung 5.102 (Nicken) / 5.31 (ja), 00:49:09.872-00:49:18.182

Michael Schmidt-Salomon: ich sagte ja $+^{\text {iteriert }}$ (.) öh ganz $\wedge$ beWUSST $\wedge{ }^{\circ} \mathrm{hh} @$ diese Werte worüber wir hier sprechen waren@(--) ne (.) ne ganz schöne Zeit lang + (-) eher $\wedge$ ÖSTliche ${ }^{\wedge}+$ als $^{\wedge}$ WESTliche $^{\wedge}$ Werte + 

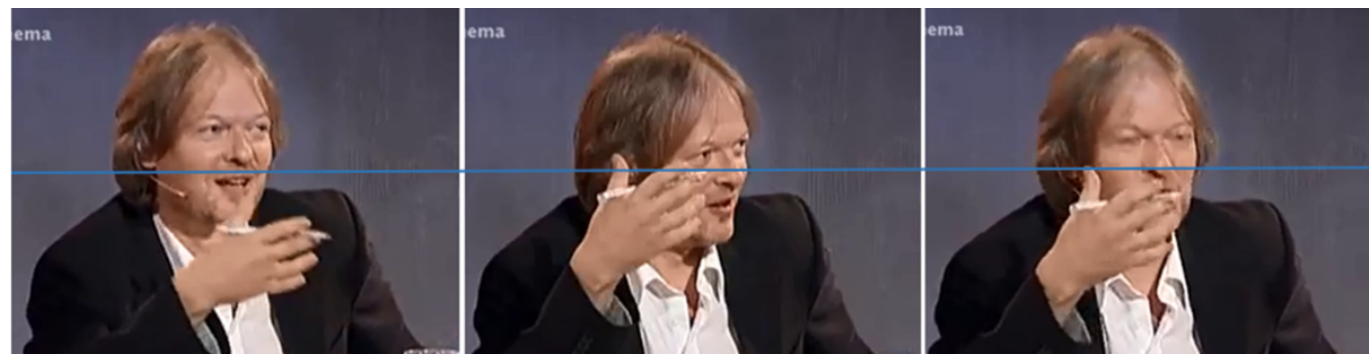

Abbildung 9: Nicken mit ja (zu Ausschnitt 16)

\subsubsection{Beat und natürlich}

Eine ziemlich starke Tendenz zur Kookkurrenz ist ebenso für den Beat und das Adverb natürlich festzustellen (5,64 \% für den Beat und 33,78 \% für natürlich). Das nachfolgende Beispiel aus der Islamdebatte veranschaulicht, wie diese evidentheitsmarkierende Kombination im Korpus aussieht. Für Frau Schwan ist es evident, dass die Gewissensfreiheit und der Glauben nicht intolerant praktiziert werden dürfen. Sie benutzt einen Beat und das Adverb natürlich um diese Selbstverständlichkeit ihrer Aussage hervorzuheben.

17. Äußerung 5.40-42 (Beat)/5.5 (natürlich), 00:16:08.819-00:16:20.604

Gesine Schwan: ich MEIne also ${ }^{\circ} \mathrm{h}$ dass wir die QUELlen (--) unserer Gewissensfreiheit (.) unseres Glaubens nicht verLEUGnen sollen aber $\wedge^{\wedge^{\text {Kopf }}+2 \text { Hände einfach }}$ naTÜRlich $\wedge$

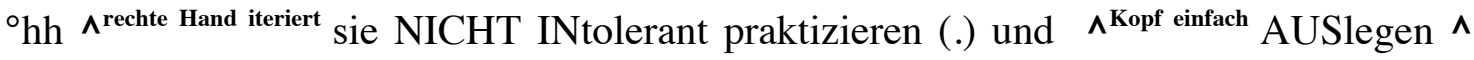
dürfen
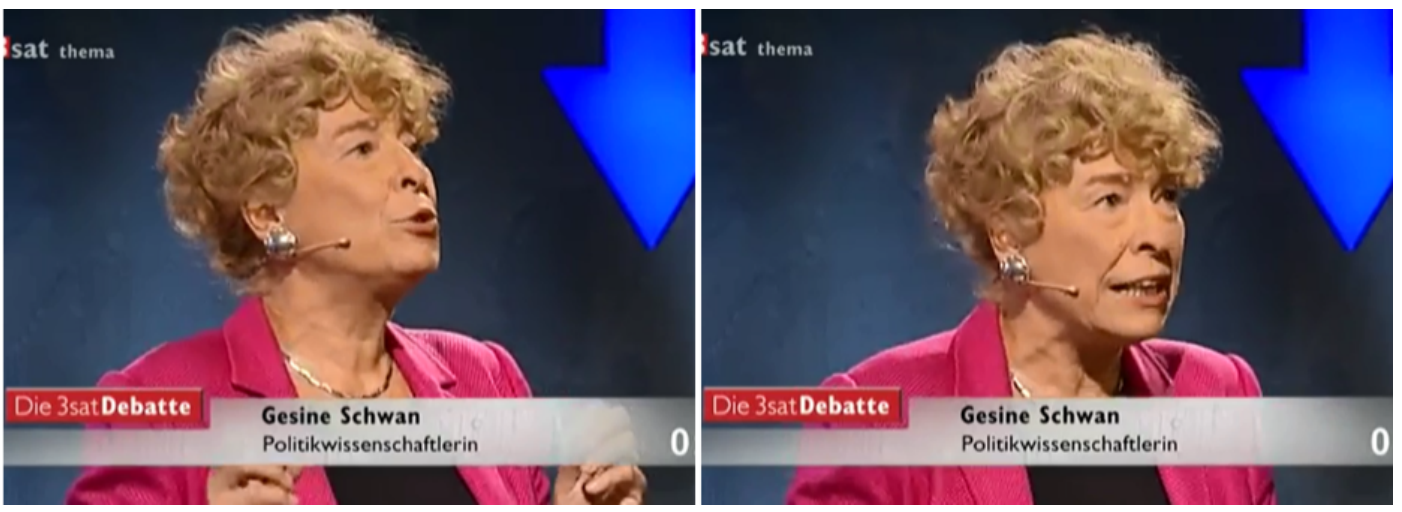

Abbildung 10: Einfacher Kopf- und Handbeat mit natürlich (zu Ausschnitt 17)

Die Abschnitte 4.2.1.1 und 4.2.1.2 haben bereits große funktionale Überlappungen zwischen dem Beat und dem Nicken aufgedeckt und demzufolge lassen sich bei der Korrelation zwischen einem Beat und natürlich die gleichen Befunde feststellen wie bei der Korrelation zwischen einem Nicken und natürlich, wenn auch in einem anderen Verhältnis: Die Bestätigung und die Bitte um Bestätigung büßen bei einem Beat etwas an Kraft ein, zugunsten der betonenden Wirkung. 


\subsubsection{Weitere Gesten mit natürlich}

Aufgrund ihrer Frequenz ist auch die Verbindung von natürlich mit Gesten wie dem Achselzucken, dem Kopfschütteln, dem Tilt, dem Heben der Augenbrauen oder dem intersubjektiven Deiktikum nennenswert. Vonseiten des Adverbs natürlich ist die jeweilige Frequenz zwar in keinem der Fälle extrem hoch, aber für die Geste liegt sie jeweils bei ca. $10 \%$. Im Vergleich zu anderen Kookkurrenzraten verdient eine solche Frequenz somit besondere Erwähnung, insbesondere weil auch die Frequenzen bei natürlich mit 8,11-28,38\% immerhin nicht zu vernachlässigen sind.

Eine Übersicht der Funktionen von natürlich in einer selbstverständlichen Aussage wurde bereits im Abschnitt 4.1.2 geliefert. Auch für das Kopfschütteln und den Tilt ist die Verbindung mit der Evidentheit schon erörtert worden (siehe Abschnitt 4.2.1.3 für das Kopfschütteln und Abschnitt 4.2.1.5 für den Tilt). Die Rolle des Achselzuckens ist ebenfalls in der einschlägigen Literatur bereits gezeigt worden (siehe 2.3). So kann es laut Debras/Cienki (2012: 936) unter anderem Selbstverständlichkeit und Notwendigkeit markieren. Die evidentheitsmarkierende Wirkung des intersubjektiven Deiktikums und des Hebens der Augenbrauen verläuft über die Markierung von oder die Suche nach Einverständnis (Schoonjans 2014: 208-209 und Ishi/Ishiguro/Hagita 2014: 242; siehe auch Abschnitt 2.3).

Der unten aufgeführte Datenausschnitt liefert ein Beispiel der Kookkurrenz eines intersubjektiven Deiktikums und natürlich. Herr Liminski ist offensichtlich nicht einverstanden mit der Behauptung, die Politik interessiere sich nicht für Jugendliche und Studierende. Die jungen Menschen seien doch beispielsweise beim Wahlkampf andauernd anwesend. Zugleich geht er auf den möglichen Vorwurf der Gesprächspartner ein, dass diese jungen Menschen ,nur als Wahlkampfgeck“ eingesetzt würden. Obwohl ihm diese These eher unwahrscheinlich scheint, weist er mit einem intersubjektiven Deiktikum und natürlich auf deren Möglichkeit hin. Er führt also einen möglichen Einwand seiner Zuhörer an, um ihn sofort wieder abzulehnen. Auffällig ist, dass die Zeigegeste hier nicht mit der flachen Hand realisiert wird, wie es für intersubjektive Deiktika üblich ist, sondern einfach mit dem ausgestreckten Zeigefinger. Inwiefern dies darauf zurückzuführen ist, dass er die These selber für unwahrscheinlich hält (er markiert auch nur deren Möglichkeit und nicht die These selbst als evident), muss an dieser Stelle dahingestellt bleiben.

18. Äußerung 1.51,00:25:14.178-00:25:17.811

Nathanael Liminski: $\wedge$ ! rechte Hand jetzt kann $\wedge$ ! man natürlich sagen die werden nur als Wahlkampfgeck ${ }^{\circ} \mathrm{hh}$ sozusagen gebraucht 


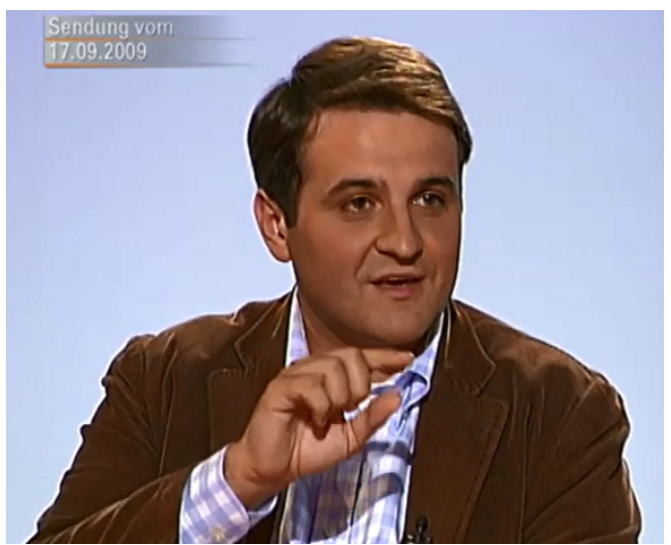

Abbildung 11: Intersubjektives Deiktikum mit natürlich (zu Ausschnitt 18)

Das Adverb kann sich aber auch mit anderen evidentheitsmarkierenden Gesten zusammentun. Im nächsten Beispiel verknüpft sich das Adverb mit einem Kopfschütteln, um anzudeuten, dass es evident ist, dass man sich überlegen muss, wie der Verkehr bei Bauarbeiten geleitet wird. Die Einzigmöglichkeit des Kopfschüttelns weist andere Möglichkeiten sofort zurück und gibt der Aussage zusammen mit dem Adverb natürlich eine selbstverständliche Wirkung.

19. Äußerung 4.22, 00:07:37.361-00:07:43.963

Elmar Keutgen: zur oberen Bergstraße hin ${ }^{\circ} \mathrm{hh}$ - ${ }^{\text {iteriert }}$ wird man natürlich $-+{ }^{\circ} \mathrm{h}+\{$ sich über den Verkehr $\left.{ }^{\circ} \mathrm{h}\right\}$ wiederum öh öh GeDANKen machen müssen

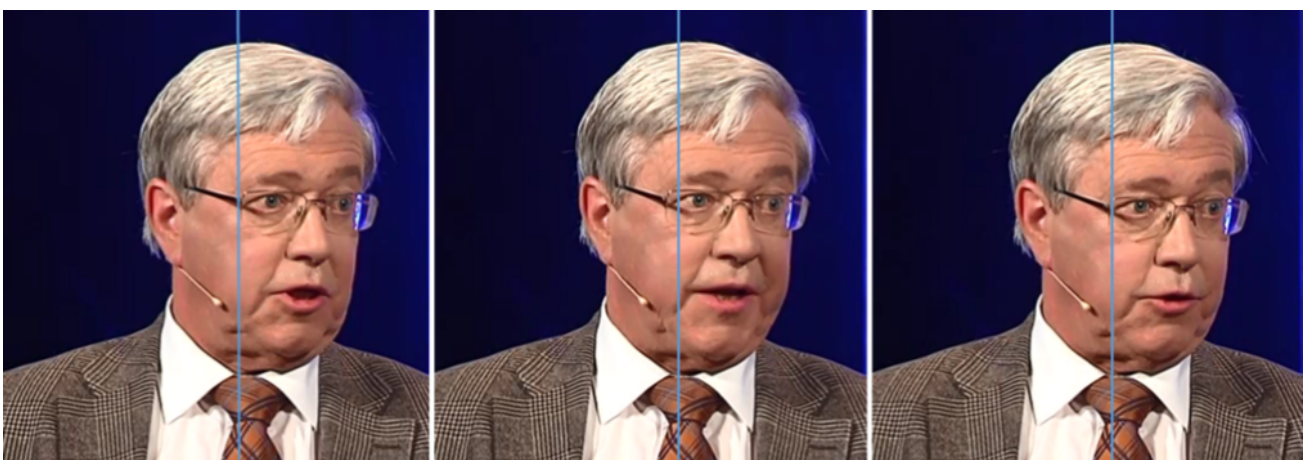

Abbildung 12: Kopfschütteln mit natürlich (zu Ausschnitt 19)

\subsubsection{Kopfschütteln und einfach}

Schoonjans (2014: 179-181) hat in seinen Daten eine starke Korrelation zwischen einfach und dem Kopfschütteln aufgedeckt, zumindest vonseiten der Partikel: 23,70 \% der Instanziierungen von einfach werden mit einem Kopfschütteln kombiniert, während 10,77 \% der Instanziierungen eines Kopfschüttelns mit der Partikel einfach einhergehen.

Die Verknüpfung von Kopfschütteln und einfach lässt sich im hier analysierten Korpus tatsächlich vorfinden, wie das nächste Beispiel zeigt. Ein Zuschauer aus dem Publikum bei der Islamdebatte äußert seine Unzufriedenheit in Bezug auf die Trennung von Moral und Religion, die von den Diskussionsteilnehmern geschaffen wird. Moral und Religion seien inhärent miteinander verwoben und es dürfte deshalb kein Staunen erregen, dass er sich gegen diese Trennung wehrt. Das Kopfschütteln und einfach verleihen der Aussage eine evidente Bedeutung, indem der Sprecher damit andere Möglichkeiten verwirft. Der Sprecher fühlt sich, sei- 
nes Glaubens wegen, dazu verpflichtet, sich gegen die Teilung von westlichen Werten und Religion zu wehren.

20. Äußerung 5.38 (Kopfschütteln)/5.23 (einfach), 00:42:35.472-00:42:40.406

Zuschauer: auch die westlichen Werte HABen IHRE BASIS in der Religion und deswegen ${ }^{\circ} \mathrm{hh}$ wehre ${ }^{\text {iteriert }}$ ich mich dagegen einfach - dass das hier alles so auseinander dividiert werden soll

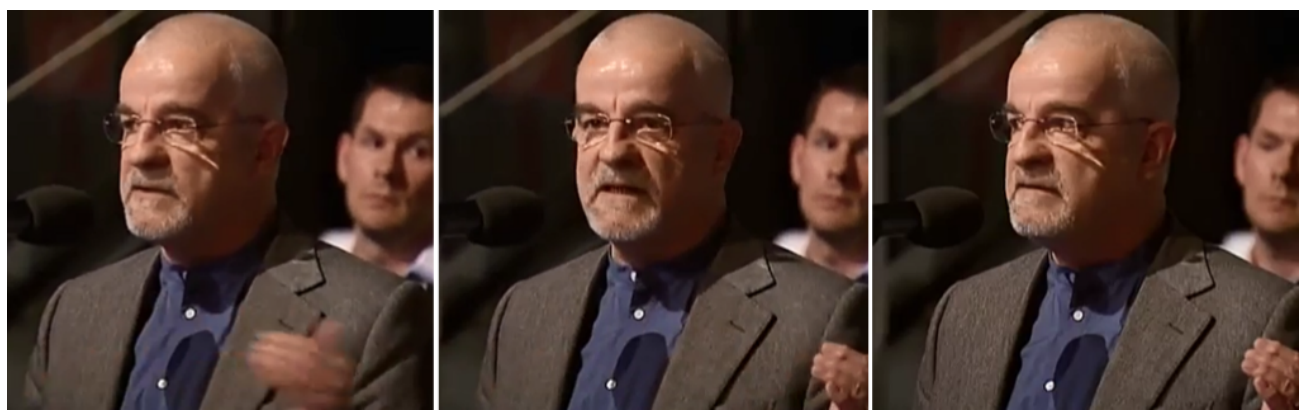

Abbildung 13: Kopfschütteln mit einfach (zu Ausschnitt 20)

Trotzdem ist das Bild, was die Korrelation zwischen Kopfschütteln und einfach angeht, in den hier analysierten Daten viel verzerrter als in Schoonjans' Daten. Die Zahl der Kombinationen für einfach steigt zwar beachtlich $(75,00 \%)$, vonseiten des Kopfschüttelns sinkt die Frequenz der Verbindung aber auf 2,27\%. Interessanterweise sind in den Daten auch Kombinationen von einem Kopfschütteln mit anderen verbalen Evidentheitsmarkierern vorhanden, die eine mit Schoonjans (2014) vergleichbare Frequenz aufweisen. Diese Aussage stimmt erstens für Kopfschütteln und natürlich (siehe auch 4.3.4) sowie für Kopfschütteln und ja. In den beiden Fällen ersetzt ein anderer verbaler Evidentheitsmarkierer die Partikel einfach. Weiterhin weisen die Kombinationen Tilt und natürlich sowie Heben der Augenbrauen und natürlich (siehe auch 4.3.4) ebenfalls ähnliche Frequenzen auf.

Die Tatsache, dass das Kopfschütteln und einfach eine deutlich weniger starke Tendenz zur Kookkurrenz aufweisen als bei Schoonjans, dürfte (unter anderem) auf die Art des Datenmaterials zurückzuführen sein. Tatsächlich hat auch Schoonjans selber bereits darauf hingewiesen, dass diese Kookkurrenz zum Beispiel in Sportberichten wesentlich häufiger vorkommt als etwa in Parlamentsreden. Die Vermutung liegt nahe, dass hier die persuasive Natur der Daten eine Rolle spielt: Ähnlich wie in Parlamentsreden versuchen die Sprecher in den für vorliegende Studie verwendeten Debatten, die Zuhörer zu überzeugen, während das zum Beispiel in Sportberichten weniger der Fall ist. Vermutlich ist dies darauf zurückzuführen, dass sowohl das Kopfschütteln als auch die Partikel einfach eher andeuten, dass es sich bei der Evidentheit um eine subjektive Einschätzung handelt (Thurmair 1989: 132 spricht im Falle von einfach von ,sprecherbezogener Evidenz'), während in persuasiven Kontexten weniger subjektiv gefärbte Elemente rhetorisch effektiver sein dürften. Dies dürfte erklären, dass in den Debatten (wie in den Parlamentsreden) die Tendenz zur Kookkurrenz von einfach und Kopfschütteln weniger stark ist bzw. dass das Kopfschütteln in den Debatten eher dazu tendiert, mit einem weniger subjektiv wirkenden Evidentheitsmarkierer (wie natürlich) aufzutreten. 


\section{5 Übereinstimmungen mit der Literatur}

Im Abschnitt 2.3 wurde bereits eine Übersicht der einschlägigen Literatur zum Thema, gestische Evidentheitsmarkierung' geliefert. Allerdings beziehen sich diese Studien nicht immer auf das Deutsche. Dementsprechend wird jetzt zum Schluss noch kurz der Frage nachgegangen, inwiefern sich die Angaben aus der Literatur auf das Deutsche ausbreiten lassen bzw. mit den Ergebnissen der vorliegenden Studie im Einklang sind.

Wesentliche Unterschiede zwischen den Ergebnissen dieser Arbeit und der bereits existierenden Literatur lassen sich vorerst nicht aufdecken. Die in der Literatur angesprochenen Gesten sind in den hier analysierten Daten vorzufinden, und die Funktionen, die sie in Evidentheitskontexten aufnehmen können, stimmen weitestgehend mit ihren Beschreibungen in der Forschungsliteratur überein. So ist das Nicken tatsächlich mit Einstimmung (Whitehead 2011) und Bestätigung (Schoonjans 2014) zu verknüpfen, das Achselzucken kann mit Selbstverständlichkeit verknüpft werden (Debras/Cienki 2012), das Kopfschütteln funktioniert als eine intensivierende Geste und kann „Ausnahmslosigkeit“ (Schoonjans 2014: 177) markieren, und der ,Palm lateral', der ,horizontal Palm‘ und der Beat sind ebenfalls in Evidentheitskontexten belegt.

Die Markierung von „Affiliation“, die Debras/Cienki (2012: 935) dem Tilt zuschreiben, und die vergewisserungssignalähnlichen Funktionen der Hebung der Augenbrauen (Jehoul 2014) können im Rahmen dieser Arbeit nicht ohne Weiteres bestätigt werden. Allerdings ist ihre Verhaltensweise mit diesen Befunden nicht inkompatibel.

Eine eingehende Untersuchung der letzten noch nicht besprochenen Geste, des intersubjektiven Deiktikums, würde den Rahmen dieser Arbeit sprengen. Auffällig ist jedoch, dass die deiktischen Gesten im Allgemeinen im Korpus nicht sehr häufig zur Markierung von Evidentheit eingesetzt werden (5,37\% der Evidentheitsfälle). Zu bemerken ist zudem, dass die Funktion des intersubjektiven Deiktikums durchaus in den Conduit integriert wird. Folglich wird der Conduit häufig in Kombination mit einem intersubjektiven Deiktikum verwendet, um Einverständnis zu markieren oder nach Einverständnis zu suchen (vgl. Schoonjans 2014: 208-209). Auf die referentiellen Deiktika trifft diese Beobachtung in der Regel nicht zu, weil sie schlichtweg auf ein Objekt zeigen. Ihr Ziel ist es, ein Objekt, auf das während der Äußerung hingewiesen wird, für den Gesprächspartner zu veranschaulichen ohne dabei auch Einverständnis seitens des Gesprächspartners einzuholen.

\section{Schlussfolgerungen und Ausblick}

In diesem Beitrag wurde die multimodale Markierung von Evidentheit im Deutschen untersucht. Die Ergebnisse dieser Untersuchung stimmen durchaus mit dem aktuellen Forschungsstand (2.3) überein (5). Fokussiert wurden die häufigsten verbalen und gestischen Evidentheitsmarkierer sowie deren Kookkurrenzverhalten. Bei den verbalen Elementen handelt es sich um ja, natürlich, doch, klar und eben, während als die frequentesten evidentheitsliierten Gesten Beats, Nicken, Kopfschütteln, Conduit-Gesten und Tilts aufgedeckt wurden. Während sich die verbalen Evidentheitsmarkierer nur selten unter-einander kombinieren, konnte gezeigt werden, dass Kombinationen der betroffenen Gesten durchaus geläufig sind. Das Vorkommen eines gestischen Evidentheitsmarkierers alleine ist dagegen wiederum eher selten. 
Der Hauptteil dieser Arbeit bestand in der genaueren Betrachtung der Verknüpfungen von gestischen und verbalen Evidentheitsmarkierern. In diesem Zusammenhang sind vier frequente Verbindungen besprochen worden: die Kombination von Nicken und natürlich, von Nicken und ja, von Beat und natürlich und von einer evidentheitsmarkierenden Geste (Achselzucken, Kopfschütteln, Tilt, Heben der Augenbrauen oder intersubjektivem Deiktikum) und natürlich. Aufgrund der Feststellung von Schoonjans (2014), dass die Kookkurrenz von Kopfschütteln und einfach im Deutschen bemerkenswert ist, wurde die Häufigkeit dieser Verknüpfung auch für diese Datensammlung untersucht. Da die Frequenz der Verbindung nur vonseiten des verbalen Evidentheitsmarkierers einfach beachtenswert war, war das Ergebnis nicht völlig mit Schoonjans' Ergebnissen im Einklang. Daraufhin wurde die Hypothese erstellt, dass die mehr oder weniger persuasive Natur der Daten auf die Distribution der Evidentheitsmarkierer einen Einfluss haben dürfte. Weitere Forschung ist hier allerdings erforderlich.

Zudem ist anzumerken, dass in vorliegender Arbeit auf nonverbaler Seite lediglich die Gestik untersucht wurde. Allerdings dürften auch weitere nonverbale Ausdrucksebenen im Bereich der Evidentheit eine Rolle spielen. Auf die Bewegungen der Augenbrauen wurde oben bereits hingewiesen, aber es ist nicht auszuschließen, dass auch Faktoren wie Blickverhalten und Körperhaltung einen Beitrag zur Evidentheitsmarkierung leisten. Diese Erweiterung gilt es allerdings noch zu untersuchen, genauso wie die Interaktion zwischen diesen Ausdrucksebenen untereinander sowie mit der verbalen und gestischen Ebene.

Darüber hinaus wäre es interessant, der Frage nachzugehen, inwiefern es sich bei den aufgedeckten Kookkurrenzmustern um multimodale Konstruktionen im Sinne von Schoonjans/Brône/Feyaerts (2015) handelt. Tatsächlich konnte gezeigt werden, dass bestimmte verbale und gestische Evidentheitsmarkierer häufiger gemeinsam eingesetzt werden und durchaus eng miteinander verwoben sind und zusammenwirken. Ob und inwiefern davon auszugehen ist, dass die beiden Ausdrucksebenen in einer multimodalen Konstruktion integriert sind oder doch vielmehr als separate Konstruktionen nebeneinander bestehen, ist allerdings noch ein Forschungsdesiderat.

Zum Schluss ist noch auf Abschnitt 2.2 zurückzuverweisen. In diesem Abschnitt wurde bereits darauf hingewiesen, dass ,Evidentheit“ ein nur schwer genau zu bestimmender Begriff ist, sodass in vorliegender Arbeit von einem breiten Evidentheitsbegriff ausgegangen wurde. Diesbezüglich wurde die Frage gestellt, ob bzw. inwiefern die Analyse der Gesten zu einem besseren Verständnis von Evidentheit führen könnte und so erlauben würde, den Begriff auch im Hinblick auf die Ebene des Verbalen weiter auf den Punkt zu bringen. Auch wenn bei solchen Fragen immer die Gefahr des Zirkelschlusses besteht (wie entscheidet man, welche Gesten bei einer Analyse von Evidentheit einzubeziehen sind, wenn man keinen genau abgegrenzten Evidentheitsbegriff hat?), kann das Zusammenführen unterschiedlicher Ausdrucksebenen (in diesem Fall die verbale und die gestische) tatsächlich zu einem besseren Verständnis des Evidentheitsbegriffs beitragen und somit einen wichtigen Schritt zum Etablieren der Evidentheit als (sprachübergreifende) funktionale Kategorie darstellen. Aufgrund ihres beschränkten Umfangs konnte die vorliegende Untersuchung diesem Ziel allerdings nicht völlig gerecht werden; auch in dieser Hinsicht ist also noch weitere Forschung notwendig um der Kategorie weiter auf den Grund zu gehen. 


\section{Korpus}

Das TV-Duell (2009): "Merkel-Steinmeier". Leitung: Frank Plasberg, Peter Limbourg, Maybrit Illner und Peter Klöppel. ARD, ZDF, RTL, Sat1 und Phoenix.

Die 3sat Debatte (2012): „Der Islam passt zu unseren westlichen Werten!“. Leitung: Theo Koll. 3SAT.

Markus Lanz (2011): Leitung: Markus Lanz. ZDF. www.youtube.com/watch?v= jOolAfjv3xg [20.02.2017].

Talksendung des Parlamentsfernsehens (2009): „Zukunftsperspektiven junger Leute in der Wirtschaftskrise“. Leitung: Katrin Prüfig. Parlamentsfernsehen des Deutschen Bundestages. www.bundestag.de/mediathek?videoid=324906\#url=L211ZGlhdGhla292ZXJsYXk $=\& \bmod =$ mediathek $[10.02 .2017]$.

Wahlen 2012 (2012): „Kommunalwahlen in Eupen“. Leitung: Adrian Küchenberg. BRF.

\section{Literatur}

Bednarek, Monika (2006): "Epistemological positioning and evidentiality in English news discourse. A text-driven approach". Text \& Talk 26/6: 635-660.

Bressem, Jana/Müller, Cornelia (2014): “A repertoire of German recurrent gestures with pragmatic functions". In: Müller, Cornelia et al. (eds.): Body - Language - Communication. An international handbook on multimodality in human interaction. Vol. 2. Berlin/Boston, Mouton de Gruyter: 1575-1591. (= HSK 38.2).

Bublitz, Wolfram (1978): Ausdrucksweisen der Sprechereinstellung im Deutschen und Englischen. Untersuchungen zur Syntax, Semantik und Pragmatik der deutschen Modalpartikeln und Vergewisserungsfragen und ihrer englischen Entsprechungen. Tübingen: Max Niemeyer.

Calbris, Geneviève (2011): Elements of meaning in gesture. Amsterdam/Philadelphia: Benjamins.

Debras, Camille/Cienki, Alan (2012): "Some uses of head tilts and shoulder shrugs during human interaction, and their relation to stancetaking". In: SocialCom/PASSAT'12 - Proceedings of the 2012 ASE/IEEE International Conference on Social Computing and 2012 ASE/IEEE International Conference on Privacy, Security, Risk and Trust. Los Alamitos/CA, IEEE Computer Society: 932-937.

Dendale, Patrick/Tasmowski, Liliane (2001): "Introduction. Evidentiality and related notions". Journal of Pragmatics 33/3: 339-348.

Duden online. Duden Verlag. www.duden.de [27.01.2015].

Franck, Dorothea (1980): Grammatik und Konversation. Königstein im Taunus: Scriptor.

Fricke, Ellen (2012): Grammatik Multimodal. Wie Wörter und Gesten zusammenwirken. Berlin: de Gruyter.

Holler, Judith (2010): "Spreaker's use of interactive gestures as markers of common ground". In: Kopp, Stefan/Wachsmuth, Ipke (eds.): Gesture in embodied communication and human computer interaction. Berlin, Springer: 11-22.

Ishi, Carlos Toshinori/Ishiguro, Hiroshi/Hagita, Norihiro (2014): “Analysis of relationship between head motion events and speech in dialogue conversations". Speech Communication 57: 233-243. 
Jehoul, Annelies (2014): Multimodale uitdrukkingen van vanzelfsprekendheid. Een empirische corpusstudie. Unveröffentlichte Diplomarbeit, KU Leuven Faculteit Letteren.

Kendon, Adam (2002): "Some uses of the head shake". Gesture 2/2: 147-182.

Kendon, Adam (2004): Gesture. Visible action as utterance. Cambridge: Cambridge University Press.

McClave, Evelyn (2000): "Linguistic functions of head movements in the context of speech". Journal of Pragmatics 32/7: 855-878.

McNeill, David (1992): Hand and mind. What gestures reveal about thought. Chicago: University of Chicago press.

McNeill, David (2005): Gesture and thought. Chicago: University of Chicago Press.

Müller, Cornelia (1996): „Zur Unhöflichkeit von Zeigegesten“. Osnabrücker Beiträge zur Sprachtheorie 52: 196-222.

Müller, Cornelia (2004): "Forms and uses of the palm up open hand. A case of a gesture family?’. In: Müller, Cornelia/Posner, Roland (eds.): The semantics and pragmatics of everyday gestures. Berlin, Weidler: 233-256.

Müller, Cornelia (2010): „Wie Gesten bedeuten. Eine kognitiv-linguistische und sequenzanalytische Perspektive“. Sprache und Literatur 105: 37-68.

Müller, Cornelia (2014): "Gesture as 'deliberate expressive movement'”. In: Seyfeddinipur, Mandana/Gullberg, Marianne (eds.): From gesture in conversation to visible action as utterance. Amsterdam/Philadelphia, Benjamins: 127-151.

Norris, Sigrid (2004): Analyzing multimodal interaction. A methodological framework. London: Routledge.

Oxford English Dictionary online. Oxford University Press. www.oed.com [27.01.2015].

Rinas, Karsten (2007): „Bekanntheit? Begründung? Einigkeit? Zur semantischen Analyse der Abtönungspartikel ja“. Deutsch als Fremdsprache 44: 205-211.

Robert, Paul (2011): Le petit Robert. Dictionnaire alphabétique et analogique de la langue française. Paris: Le Robert.

Selting, Margret et al. (2009): „Gesprächsanalytisches Transkriptionssystem 2 (GAT2)“. Gesprächsforschung 10: 353-402.

Schoonjans, Steven (2014): Modalpartikeln als multimodale Konstruktionen. Eine korpusbasierte Kookkurrenzanalyse von Modalpartikeln und Gestik im Deutschen. Unveröffentlichte Dissertation, KU Leuven Faculteit Letteren.

Schoonjans, Steven/Brône, Geert/Feyaerts, Kurt (2015): „Multimodalität in der Konstruktionsgrammatik. Eine kritische Betrachtung illustriert anhand einer Gestikanalyse der Partikel einfach“. In: Bücker, Jörg/Günthner, Susanne/Imo, Wolfgang (eds.): Konstruktionsgrammatik V. Konstruktionen im Spannungsfeld von sequenziellen Mustern, kommunikativen Gattungen und Textsorten. Tübingen, Stauffenburg: 291-308.

Thurmair, Maria (1989): Modalpartikeln und ihre Kombinationen. Tübingen: Max Niemeyer.

Waltereit, Richard (2006): Abtönung. Zur Pragmatik und historischen Semantik von Modalpartikeln und ihren funktionalen Äquivalenten in romanischen Sprachen. Tübingen: Max Niemeyer.

Whitehead, Kevin Andrew (2011): "Some uses of head nods in 'third position' in talk-ininteraction". Gesture 11/2: 103-122. 


\section{Anhang A: Transkriptions- und Annotationskonventionen}

\section{Transkription der Gestik}

Da die vorliegende Studie auf der Arbeit Jehouls (2014) aufbaut, wurde für die Andeutung der Gesten in den Transkripten eine Variante ihres Transkriptionssystems verwendet. Die benutzten Angaben sind unten aufgelistet. Etwaige weiterführende Angaben zu den zur Diskussion stehenden Gesten sind im Superscript aufgenommen.

[ ] Conduit

++ Nicken

- - Kopfschütteln

\{\}$\quad$ Tilt

$\wedge \wedge$ Beat

$<>$ horizontal Palm

$>\quad$ Palm lateral

/ 1 Achselzucken

$* * \quad$ Augenbrauen heben

! ! intersubjektives Deiktikum

@@ referentielles Deiktikum

\section{Transkription der gesprochenen Sprache}

Für die Transkription der verbalen Ebene wurden einzelne Elemente der GAT2-Konvention (Selting u. a. 2009) herangezogen:

(.) Pause bis ca. 0,2 Sekunden

(-) Pause von ca. 0,2 - 0,5 Sekunden

(--) Pause von ca. $0,5-0,8$ Sekunden

(--) Pause von ca. $0,8-1,0$ Sekunden

(4) Pause von ca. 4 Sekunden

${ }^{\circ} \mathrm{h} \quad$ hörbares Einatmen von ca. 0,2 - 0,5 Sekunden

${ }^{\circ} \mathrm{hh} \quad$ hörbares Einatmen von ca. 0,5 - 0,8 Sekunden

${ }^{\circ} \mathrm{hhh} \quad$ hörbares Einatmen von ca. 0,8 - 1 Sekunden

Großschreibung auffällige Betonung der Silbe

\section{Anhang B: Das Videokorpus}

Das erste Video ist eine politische Debatte zwischen Sarah Jermutus, Pawel Hörnle, Nathanael Liminski und Norbert Lammert, moderiert von Katrin Prüfig. Diese Debatte wurde am 17. September 2009 im Parlamentsfernsehen des Deutschen Bundestages ausgestrahlt. Das Thema ist der Einfluss der Politik auf das Glück der Studierenden, sowohl in der Karriere als auch im Leben im Allgemeinen.

Im zweiten Video wird die Zuerkennung des Bambi für Integration an Bushido in Frage gestellt und diskutiert. Die Teilnehmer dieser Diskussion sind Bushido selbst, Peter Maffay, Richard David Precht, Sido und Gaby Decker. Auch der Moderator Markus Lanz nimmt an 
der hitzigen Debatte teil. Die Sendung ist eine Produktion des ZDF und wurde am 23. November 2011 gesendet. Für die Analyse wurde ein Ausschnitt von 17 Minuten verwendet.

Das dritte Video ist ein zehnminütiger Ausschnitt des TV-Duells zwischen Angela Merkel und Frank-Walter Steinmeier während des Wahlkampfes 2009. Dieses Duell wird von vier Moderatoren (Frank Plasberg, Peter Limbourg, Maybrit Illner und Peter Klöppel) geleitet. Die Debatte wurde am 13. September 2009 live in der ARD übertragen.

Das vierte Video ist ebenfalls eine Wahldebatte unter Begleitung des Moderators Adrian Küchenberg. Die vier Teilnehmer dieser Debatte (Claudia Niessen, Werner Baumgarten, KarlHeinz Klinkenberg und Elmar Keutgen) kandidieren alle für das Bürgermeisteramt der Stadt Eupen. Diese Wahldebatte wurde am 5. Oktober 2012 im BRF (Belgischen Rundfunk) ausgestrahlt.

Das letzte Videogespräch bezieht sich auf die Frage, inwiefern die westlichen Werte und der Islam zu versöhnen sind. Diese Diskussion zwischen Michael Schmidt-Salomon, Gesine Schwan, Hamed Abdel-Samad und Tarafa Baghajati moderierte Theo Koll. Die Debatte, eine 3SAT-Produktion, wurde am 27. August 2012 ausgestrahlt. 